Pacific Journal of Mathematic 


\title{
ON GENERALIZED RESOLVENTS AND $Q$-FUNCTIONS OF SYMMETRIC LINEAR RELATIONS (SUBSPACES) IN HILBERT SPACE
}

\author{
H. LANGER AND B. TeXtorius
}

Dedicated to M. G. Krein on the occasion of his 70th birthday

The notion of a $Q$-function and M. G. Krein's formula for the generalized resolvents of a symmetric operator are extended to the case of a symmetric linear relation. Applications to linear non-densely defined bounded operators as well as to semibounded linear relations are given.

In some problems related to the spectral theory in Hilbert space it is more natural and at the same time often less restrictive to use symmetric linear relations (in the terminology of [1], subspaces in the terminology of [2-4]) instead of symmetric operators. Hence the question arises if the theory of generalized resolvents of symmetric operators can be extended to symmetric linear relations. In [4] a description of all generalized resolvents of a symmetric linear relation was given, following the lines of A. V. Straus [5] in the operator case. It is the aim of this paper to generalize M. G. Kreinn's formula for the generalized resolvents of a symmetric operator (see [6,7]) to the symmetric linear relation case. This can be done rather easily by means of the Cayley transformation, using the results of [8]. However, in this connection there arise natural problems and questions: To introduce and to study the $Q$-function of a linear relation, to prove criteria for the selfadjoint extension of the given symmetric linear relation being an operator, to study the special case of a bounded nondensely defined operator etc.

After the necessary definitions and their simple consequences in $\S 1$, the $\S 2$ is devoted to a study of the $Q$-function. From arguments similar to those in $[9,10]$ it follows that every function $Q$, whose values are bounded operators in a Hilbert space and which is holomorphic in the upper half plane and has the property

$$
\operatorname{Im} Q(z) \gg 0 \text { if } \operatorname{Im} z>0
$$

is a $Q$-function of a symmetric linear relation. Let us remind here that in the operator case besides $(*)$ additional conditions appear (see [9]). In $\S 3$ the generalization of M. G. Kreinn's formula to symmetric linear relations is proved. In terms of the $Q$-function a necessary and sufficient condition for a minimal selfadjoint linear relation extension 
to be an operator follows. In $\S 4$ we give a description of all generalized selfadjoint contraction resolvents of a symmetric nondensely defined contraction $T$, which is equivalent to a recent result of M. G. Kreǐn and I. E. Ovčarenko [11, 12]. Moreover, we consider those generalized resolvents of $T$, which correspond to minimal selfadjoint extensions having their spectra in an interval $[\alpha, \beta],-\infty \leqq \alpha \leqq-1$, $1 \leqq \beta \leqq \infty$. As an application some statements about nonnegative linear relations are proved, which complete corresponding results of E. Coddington $[2]^{1}$.

Finally we mention that some statements of this paper can be generalized to the case of unequal defect numbers (for the operator case see e.g., [14]) as well as to symmetric linear relations in a $\Pi_{\kappa^{-}}$ space, which will be considered elsewhere.

This paper ia dedicated to Professor M. G. Krein, to whom we are obliged not only for many inspiring ideas in his works and personal communications, but also for our acquaintance.

1. Preliminaries. 1. Let $\mathfrak{S}$ be a Hilbert space. A closed linear relation (c.l.r.) in $\mathfrak{S}$ is a (closed) subspace of $\mathfrak{S}^{2}=\mathfrak{S} \oplus \mathfrak{S}$ (see [2], [3], [4]). Evidently, the graph of a closed linear operator in $\mathfrak{S}$ is a c.l.r. $T$ in $\mathfrak{S}$ with the property that

$$
\{0, g\} \in T \text { implies } g=0,
$$

and, vice versa, a c.l.r. $T$ with this property is the graph of a closed linear operator in $\mathfrak{S}$. In the following we therefore identify closed linear operators and c.l.r. with the property (1.1), that is, instead of $\{f, g\} \in T$ in this case we shall also write $g=T f$. If $T$ is a c.l.r. in $\mathfrak{S}$ we put

$$
\begin{aligned}
\mathfrak{D}(T): & =\{f:\{f, g\} \in T\}, \Re(T):=\{g:\{f, g\} \in T\}, \\
T(f): & =\{g:\{f, g\} \in T\} .
\end{aligned}
$$

The sum of two c.l.r. and the product of a c.l.r. with a complex number are defined in the usual way (see e.g., [2]). The inverse of a c.l.r. $T$ is the c.l.r.

$$
T^{-1}:=\{\{g, f\}:\{f, g\} \in T\}
$$

and the adjoint $T^{*}$ of $T$ is defined by

$$
T^{*}:=\{\{h, k\}:(g, h)=(f, k) \text { for all }\{f, g\} \in T\} \text {. }
$$

Two c.l.r. $T$ in $\mathscr{S}$ and $T^{\prime}$ in $\mathfrak{S}^{\prime}$ are called unitarily equivalent if there exists an isometric mapping $V$ of $\mathfrak{F}$ onto $\mathfrak{S E}^{\prime}$ such that $\{V x, V y\} \in$ $T^{\prime}$ if and only if $\{x, y\} \in T$.

\footnotetext{
${ }^{1}$ In the operator case deeper results are contained in [13].
} 
Every c.l.r. $T$ in $\mathfrak{S}$ can be decomposed into a direct and orthogonal sum

$$
T=T_{0} \oplus T_{\infty},^{2}
$$

where $T_{0}$ is a closed linear operator, $\mathfrak{D}\left(T_{0}\right)=\mathfrak{D}(T)$, and $T_{\infty}:=$ $\{\{0, g\} \in T\}$.

Suppose now that the space $\mathscr{S}_{2}$ is the orthogonal sum $\mathfrak{S}_{2}=\mathscr{S}_{0} \oplus \mathscr{S}_{1}$ of two subspaces $\mathfrak{S}_{0}, \mathfrak{S}_{1}$ with corresponding orthogonal projectors $P_{0}, P_{1}$ resp. and that the c.l.r. $T$ in $\mathscr{S}$ has the form $T=T_{0} \oplus T_{\infty}$, where $T_{0}$ is a c.l.r. in $\mathfrak{S}_{0}$ and $T_{\infty}=\left\{\{0, g\}: g \in \mathfrak{S}_{1}\right\}$. Let $Q$ be a bounded linear operator in $\mathfrak{S}$ with $\mathfrak{D}(Q)=\mathscr{F}$ and suppose that the inverse $\left(T_{0}+P_{0} Q P_{0}\right)^{-1}$ in $\mathscr{S}_{0}$ is an operator. Then $(T+Q)^{-1}$ is also an operator and we have

$$
(T+Q)^{-1}=\left(T_{0}+P_{0} Q P_{0}\right)^{-1} P_{0} .
$$

Indeed, $(T+Q)^{-1}=\left\{\left\{Q f_{0}+g_{0}+g_{1}, f_{0}\right\}:\left\{f_{0}, g_{0}\right\} \in T_{0}, g_{1} \in \mathscr{S}_{1}\right\}$. Now $Q f_{0}+$ $g_{0}+g_{1}=0$ implies $P_{0} Q P_{0} f_{0}+g_{0}=0$, hence $f_{0}=0$ as $\left(T_{0}+P_{0} Q P_{0}\right)^{-1}$ is an operator. Evidently $(T+Q)^{-1} g_{1}=0$,

$$
(T+Q)^{-1}=\left\{\left\{P_{0} Q P_{0} f_{0}+g_{0}+g_{1}, f_{0}\right\}:\left\{f_{0}, g_{0}\right\} \in T_{0}, g_{1} \in \mathscr{S}_{1}\right\},
$$

which is equivalent to (1.3).

2. A c.l.r. $T$ is called dissipative if

$$
\{f, g\} \in T \text { implies } \operatorname{Im}(g, f) \geqq 0,
$$

maximal dissipative if $T$ is dissipative and $T^{\prime} \supset T, T^{\prime}$ dissipative imply $T^{\prime}=T$, and symmetric if

$$
\{f, g\} \in T \text { implies } \operatorname{Im}(g, f)=0 .
$$

It is easy to see that a c.l.r. $T$ is symmetric if and only if $T \subset T^{*}$. A c.l.r. $T$ is called selfadjoint if $T=T^{*}$.

By a fundamental theorem of Arens [1], the c.l.r. $T$ is selfadjoint if and only if $T_{0}$ in the decomposition (1.2) is a selfadjoint operator in $\mathfrak{S}_{0}=\mathfrak{R}\left(T_{\infty}\right)^{\perp}$.

Let $T$ be a symmetric c.l.r. in $\mathfrak{F}$. If $A$ is a bounded selfadjoint linear operator in $\mathfrak{S}$ with $\mathfrak{D}(A)=\mathfrak{S}$ the symbol $T \geqq A$ means $(g, f) \geqq$ $(A f, f)$ for all $\{f, g\} \in T$, and $T \leqq A$ is defined in the usual way as $-T \geqq-A$. However, the writing $A \leqq T \leqq B$ with two bounded selfadjoint operators $A, B$ means that $T$ is also an operator and that these inequalities hold in the usual operator sense. A symmetric c.l.r. $T$ is called nonnegative if $T \geqq 0$.

Let $T$ be a symmetric c.l.r. in $\mathfrak{S}$. We define

${ }^{2} \oplus$ denotes the orthogonal sum in $\mathfrak{S}^{2}$. 


$$
\mathfrak{M}_{z}:=\mathfrak{R}(T-z I), \mathfrak{R}_{z}=\mathfrak{M}_{\bar{z}}^{\perp} .
$$

There exist two cardinal numbers $n_{+}$and $n_{-}$, the upper and lower defect numbers of $T$, such that we have

$$
\operatorname{dim} \mathfrak{N}_{\bar{z}}=n_{ \pm} \quad \text { for all } z \in C_{ \pm} \cdot \cdot^{3}
$$

The Cayley transform $\mathcal{E}_{z_{0}}(T)\left(z_{0} \neq \bar{z}_{0}\right)$ of the c.l.r. $T$ in $\mathfrak{F}$ is defined as

$$
V:=\mathfrak{C}_{z_{0}}(T):=\left\{\left\{g-z_{0} f, g-\bar{z}_{0} f\right\}:\{f, g\} \in T\right\},
$$

the inverse $\widetilde{\mho}_{z_{0}}$ of the Cayley transformation $\mathfrak{F}_{z_{0}}$ is given by

$$
T=\widetilde{\mho}_{z_{0}}(V):=\left\{\left\{g-f, z_{0} g-\bar{z}_{0} f\right\}:\{f, g\} \in V\right\} .
$$

If $T$ is symmetric, $V$ is an isometric operator in $\mathfrak{S}$ with $\mathfrak{D}(V)=\mathfrak{M}_{z_{0}}$, $\Re(V)=\mathfrak{M}_{z_{0}}$. The Cayley transformation $\mathfrak{E}_{z_{0}}$ establishes a one-to-one correspondence between the set of all symmetric (selfadjoint) c.l.r. $T$ in $\mathfrak{F}$ and the set of all isometric (unitary resp.) operators $V$ in $\mathfrak{F}$. If $z_{0} \in C_{-}, \mathfrak{C}_{z_{0}}$ also defines a one-to-one correspondence between the set of all dissipative (maximal dissipative) c.l.r. $T$ in $\mathfrak{F}$ and the set of all contractions $V$ (contractions $V$ with $\mathfrak{D}(V)=\mathfrak{S}$ resp.) in $\mathfrak{S}$. If $T, T^{\prime}$ are two c.l.r. in $\mathscr{S}$ and $\mathscr{S}^{\prime}(\supset \mathscr{S})$ resp. with $T \subset T^{\prime}$, then $T^{\prime}$ is called an extension of $T$. If in this case $\mathfrak{S}^{\prime}=\mathfrak{F}$, then $T^{\prime}$ is said to be a canonical extension of $T . \quad$ Evidently $T \subset T^{\prime}$ is equivalent to $\mathbb{F}_{z_{0}}(T) \subset$ $\mathfrak{C}_{z_{0}}\left(T^{\prime}\right)$. It is well known (see e.g., [4]) that every symmetric c.l.r. admits selfadjoint extensions and that it admits canonical selfadjoint extensions if and only if its defect numbers $n_{+}$and $n_{-}$are equal.

With the Cayley transformation $\mathbb{E}_{z_{0}}$ we consider the mapping

$$
z \longrightarrow \lambda(z):=\left(z-\bar{z}_{0}\right)\left(z-z_{0}\right)^{-1}
$$

of the compact complex plane into itself. An easy calculation yields

$$
V-\lambda(z) I=\left\{\left\{g-z_{0} f,\left(\bar{z}_{0}-z_{0}\right)(g-z f)\right\}:\{f, g\} \in T\right\},
$$

hence

$$
R(V-\lambda(z) I)=R(T-z I) .
$$

The spectrum $\sigma(T)$ of the selfadjoint c.l.r. $T$ is by definition the spectrum of its operator part $T_{0}$, the extended spectrum $\sigma_{e}(T)$ of $T$ is $\sigma(T)$ if $T=T_{0}$ and $\sigma(T) \cup\{\infty\}$ if $T \neq T_{0}$. With the mapping $\lambda$ introduced above we have $\lambda\left(\sigma_{e}(T)\right)=\sigma(V), \lambda(\sigma(T))=\sigma(V) \backslash\{1\}$. The resolvent set $\rho(T)$ (extended resolvent set $\rho_{e}(T)$ ) is the complement of $\sigma(T)\left(\sigma_{e}(T)\right.$ resp.).

If $T$ is a selfadjoint c.l.r. in $\mathscr{S}$ and $z \in \rho(T)$, then (1.3) implies

$$
(T-z I)^{-1}=\left(T_{0}-z I\right)^{-1} P_{0},
$$

${ }^{3} C$ denotes the complex plane, $C_{+}\left(C_{-}\right)$the open upper (lower resp.) half plane. 
where $P_{0}$ again denotes the orthogonal projector onto $\overline{\mathfrak{D}\left(T_{0}\right)}=\Re\left(T_{\infty}\right)^{\perp}$.

A subspace $\mathfrak{F}_{1}$ of $\mathfrak{S}$ is called a reducing subspace of the dissipative c.l.r. $T$ if $\mathscr{S}_{1}$ is a reducing subspace of the Cayley transform $V=$ $\mathfrak{C}_{-z_{0}}(T), \operatorname{Im} z_{0}>0$, that is if $\mathfrak{D}(V)$ decomposes as

$$
\mathfrak{D}(V)=\mathfrak{D}_{1} \oplus \mathfrak{D}_{1}^{\prime}, \mathfrak{D}_{1} \subset \mathfrak{S}_{1}, \mathfrak{D}_{1}^{\prime} \subset \mathfrak{S}_{1}^{\perp}
$$

and we have $V \mathfrak{D}_{1} \subset \mathscr{F}_{1}, V \mathfrak{D}_{1}^{\prime} \subset \mathscr{S}_{1}^{\perp}$. Denote by $V_{1}$ the operator $\left.V\right|_{\mathscr{D}_{1}}$ in $\mathfrak{S}_{1}$. Then the c.l.r. $T_{1}:=\widetilde{\mho}_{-z_{0}}\left(V_{1}\right)$ is called the c.l.r. induced by $V$ in the reducing subspace $\mathfrak{S}_{1}$.

3. The symmetric c.l.r. $T$ in $\mathscr{S}$ is called simple if

$$
\mathfrak{S}=\text { c.l.s. }\left\{\mathfrak{R}_{z}=\Re(T-\bar{z} I)^{\lrcorner}: z \neq \bar{z}\right\} .^{4}
$$

Evidently, (1.7) is equivalent to

$$
\bigcap_{z \neq \bar{z}} \mathfrak{M}_{z}=\{0\} .
$$

Since for arbitrary $z \in C$ and c.l.r. $T$ we have $\mathfrak{R}\left(T_{\infty}-z I\right)=\mathfrak{R}\left(T_{\infty}\right)$, a simple symmetric c.l.r. is an operator. Moreover, it is not difficult to see that for a simple c.l.r. $T$ in $\mathscr{F}_{\mathcal{E}}$ there is no subspace $\mathscr{S}_{1}$ in $\mathscr{S}$ which reduces $T$ and on which $T$ induces a selfadjoint operator.

Proposition 1.1. A symmetric c.l.r. $T$ in $\mathfrak{K}$ is the orthogonal sum $T=T_{\mathfrak{n}} \bigoplus T_{s}$ of a selfadjoint c.l.r. $T_{\mathfrak{M}}$ in $\mathfrak{M}:=\bigcap_{z \neq z} \mathfrak{M}_{z}$ and $a$ simple symmetric c.l.r. $T_{s}$ in $\mathfrak{M}^{\perp}$.

Proof. Choose $z_{0} \neq \bar{z}_{0}$ and consider the Cayley transform $V:=$ $\mathbb{C}_{z_{0}}(T)$. It is an isometric operator and can hence be written as the orthogonal sum of a (uniquely determined) unitary operator $V_{\mathfrak{F}^{\prime}}$ in a subspace $\mathfrak{M}^{\prime}:=\bigcap_{|\lambda| \neq 1} \Re(V-\lambda I)$ of $\mathfrak{K}$ and a simple isometric operator $V_{s}$ in $\mathfrak{M}^{\prime}$. (Here an isometric operator is called simple if there is no reducing subspace on which it induces a unitary operator.) Relation (1.4) implies $\mathfrak{M}^{\prime}=\mathfrak{M}$. The statement hence follows if we take $T_{\mathfrak{m}}$ : = $\widetilde{\mho}_{z_{0}}\left(V_{\mathfrak{m}}\right), T_{s}:=\widetilde{F}_{z_{0}}\left(V_{s}\right)$.

4. Let (\$) be a Hilbert space. By $\mathfrak{T}(\$)$ we denote the set of all functions $z \rightarrow \mathscr{T}(z)$ with the following properties:

(1) If $z \in C_{+}$then $\mathscr{T}^{-}(z)$ is a maximal dissipative c.l.r. in (S).

(2) If $z_{0} \in C_{+}$then the Cayley transform $\mathscr{Y}(z):=\mathbb{E}_{-z_{0}}(\mathscr{T}(z))$ is a holomorphic function of $z$ in $C_{+}$.

It can be shown [4] that this definition is correct, that is the property (2) is independent of the point $z_{0} \in C_{+}$.

We always assume that $\mathscr{T} \in \mathfrak{I}(\mathbb{S})$ is extended to $C_{-}$by $\mathscr{T}(\bar{z})=$ $\mathscr{T}(z)^{*}\left(z \in C_{+}\right)$. The domain of holomorphy $\mathscr{D}_{\mathscr{T}}$ of $\mathscr{T}$ consists by

${ }^{4}$ c.l.s. $=$ closed linear span. 
definition of $C_{+} \cup C_{-}$as well as of those intervals of the real axis on which $\mathscr{V}$ has unitary boundary values $\mathscr{V}(x)=\lim _{z \in C_{+}, z \rightarrow x} \mathscr{V}(z)$, $x \in \mathscr{D}_{\mathscr{T}} \cap R^{1}$.

We put

$$
\mathscr{T}(x):=\mathfrak{F}_{-z_{0}}(\mathscr{V}(x)) \quad\left(x \in \mathfrak{D}_{\mathscr{T}} \cap R^{1}\right) .
$$

Evidently $\mathscr{T}(x)$ is a selfadjoint c.l.r.

By $\mathfrak{I}_{0}(\mathbb{S})$ we denote the subset of $\mathfrak{T}(\mathbb{S})$ consisting of all $\mathscr{T} \in \mathfrak{T}(\mathbb{S})$ which are independent of $z$. Then evidently $\mathscr{D}_{\mathscr{T}}=C$ and $\mathscr{T}(z)=$ $\mathscr{T}$ is a selfadjoint c.l.r. in $(S)$.

Proposition 1.2 (see $[7, \S 4.3])$. If $\mathscr{T} \in \mathfrak{I}(\mathbb{S})$, then there exists a decomposition $\mathbb{B}=\mathbb{S}_{0} \oplus \mathbb{S}_{\infty}$ of the space $\mathbb{B}$ with the following properties:

(1) For all $z \in \mathscr{D}_{\mathscr{F}}, \mathbb{B}_{0}$ and $\mathbb{S}_{\infty}$ are reducing subspaces of $\mathscr{T}(z)$.

(2) If $\operatorname{Im} z>0$, then the induced c.l.r. $\mathscr{T}(z)_{0}$ in $\mathbb{S}_{0}$ is a maximal dissipative operator and the induced c.l.r. $\mathscr{T}(z)_{\infty}$ in $\mathbb{S}_{\infty}$ is

$$
\mathscr{T}(z)_{\infty}=\left\{\{0, g\}: g \in \mathbb{S}_{\infty}\right\} \text {. }
$$

Indeed, choose $z_{0} \in C_{+}$and consider the Cayley transform $\mathscr{T}(z)=$ $\mathbb{E}_{-z_{0}}(\mathscr{T}(z))$. It is a holomorphic and contraction valued function if $z \in \mathscr{D}_{\mathscr{\sigma}} \cap\left(C_{+} \cup R^{1}\right)$. Hence by the maximum modulus principle the set of fixed vectors of $\mathscr{C}(z)$, that is the set of solutions $f$ of $\mathscr{V}(z) f=$ $f$, is independent of $z$ if $z \in \mathfrak{D}_{\mathscr{T}} \cap\left(C_{+} \cup R^{1}\right)$. Now the statement follows without difficulty.

Let $\mathscr{T} \in \mathfrak{T}(\mathbb{S}), z \in \mathfrak{D}_{\mathcal{F}}$ and suppose $Q$ is a bounded linear operator such that the inverse $\left(P_{0} Q P_{0}+\mathscr{T}(z)_{0}\right)^{-1}$ is also an operator. As a consequence of (1.3) we then have

$$
(Q+\mathscr{T}(z))^{-1}=\left(P_{0} Q P_{0}+\mathscr{T}(z)_{0}\right)^{-1} P_{0},
$$

where $P_{0}$ again denotes the orthogonal projector onto $\mathbb{S}_{0}$.

2. The Q-function of a symmetric c.1.r. 1 . Let $S$ be a symmetric c.l.r. in $\mathfrak{S}$ with equal (finite or infinite) defect numbers $n_{+}=$ $n_{-}=: n(>0)$. We choose a Hilbert space \&s of dimension $n$. The scalar product in $(S)$ will be denoted by the same symbol $(\cdot, \cdot)$ as the scalar product in $\mathfrak{S}$. Let $\dot{S}$ be a canonical selfadjoint extension of $S$. By $\Gamma$. we denote a function on $\rho(\stackrel{S}{)})$ with values in $[\mathfrak{S}, \mathfrak{S}]^{5}$ and the following properties:

(1) $\Gamma_{z}$ maps $(S)$ one-to-one and continuously onto $\mathfrak{R}_{z}\left(z \in \rho\left(S^{\circ}\right)\right)$,

(2) $z, \zeta \in \rho\left(S_{)}\right)$implies

${ }^{5}[\mathfrak{S}, \mathfrak{S}]$ is the set of all bounded linear operators from $\mathfrak{S}$ into $\mathfrak{S},[\mathfrak{S}, \mathfrak{\mathfrak { F }}]=:[\mathfrak{G}]$. 


$$
(z-\zeta)^{-1}\left(\Gamma_{z}-\Gamma_{\zeta}\right)=(\dot{S}-z I)^{-1} \Gamma_{\zeta}=(\dot{S}-\zeta I)^{-1} \Gamma_{z} .
$$

From (2) it follows that after fixing a $z_{0} \in \rho\left(S^{\circ}\right)$ we have

$$
\Gamma_{z}=\left(I+\left(z-z_{0}\right)(\stackrel{\circ}{S}-z I)\right)^{-1} \Gamma_{z_{0}} \text {. }
$$

LEMma 2.1. If $z_{0} \in \rho\left(S^{\circ}\right)$ and $\Gamma_{z_{0}}$ is an arbitrary one-to-one mapping from (S) onto $\mathfrak{N}_{z_{0}}$, then the function $\Gamma$. in (2.1) has properties (1) and (2). Moreover, if $S$ and $S$ are bounded operators and $\Gamma$ is a mapping from $\&$ onto $\mathfrak{R}=\mathfrak{D}(S)^{\perp}$, we have

$$
\Gamma_{z}=(\dot{S}-z I)^{-1} \Gamma \text {. }
$$

Proof. Property (2) of $\Gamma_{z}$ is obvious from (2.1) and [4, Lemma 2.4]. If $\xi \in \mathbb{S}$ and $h \in \mathfrak{M}_{\bar{z}}$, that is $h=g-\bar{z} f$ with a certain $\{f, g\} \in$ $S$, we have $(\stackrel{\circ}{S}-\bar{z} I)^{-1}(g-\bar{z} f)=f$ and

$$
\begin{aligned}
\left(\Gamma_{z} \xi, h\right) & =\left(\left(\Gamma_{z_{0}}+\left(z-z_{0}\right)(\dot{S}-z I)^{-1} \Gamma_{z_{0}}\right) \xi, g-\bar{z} f\right) \\
& =\left(\Gamma_{z_{0}} \xi, g-\bar{z} f+\left(\bar{z}-\bar{z}_{0}\right)(\dot{S}-\bar{z} I)^{-1}(g-\bar{z} f)\right. \\
& =\left(\Gamma_{z_{0}} \xi, g-\bar{z}_{0} f\right)=0,
\end{aligned}
$$

since $g-\bar{z}_{0} f \in \mathfrak{M}_{\overline{z_{0}}}=\mathfrak{M}_{z_{0}}^{\perp}$.

In order to see that $\Gamma_{z}$ is onto, take $f \in \mathfrak{R}_{z}$ and consider $f_{0}:=$ $\left(I+\left(z_{0}-z\right)\left(\stackrel{\circ}{S}-z_{0} I\right)^{-1}\right) f$. Then a simple modification of the previous argument yields $f_{0} \in \mathfrak{N}_{z_{0}}$, that is $f_{0}=\Gamma_{z_{0}} \xi$ with a (unique) $\xi \in(\mathbb{S}$ and

$$
\Gamma_{z} \xi=\left(I+\left(z-z_{0}\right)(\stackrel{\dot{S}}{\xi}-z I)^{-1}\right) \Gamma_{z_{0}} \xi=\left(I+\left(z-z_{0}\right)(\dot{S}-z I)^{-1}\right) f_{0}=f .
$$

The injectivity of $\Gamma_{z}$ follows from (1.6), the last statement is a consequence of the fact that for bounded operators $S$ and $\dot{S}$ the operator $\left(\stackrel{S}{S}-z_{0} I\right) \Gamma_{z_{0}}$ maps (S) onto $\mathfrak{D}(S)^{\perp}$.

The adjoint $\Gamma_{z}^{*}$ of $\Gamma_{z}$ is the mapping from $\mathscr{S}$ into $\mathbb{S}$ defined by the relation

$$
\left(\Gamma_{z} \xi, f\right)=\left(\xi, \Gamma_{z}^{*} f\right) .
$$

This implies $\Gamma_{z}^{*} \mathfrak{M}_{\bar{z}}=\{0\}$, and $\Gamma_{z}^{*}$ maps $\mathfrak{N}_{z}$ bijectively onto $\mathbb{S}$.

2. With the function $\Gamma$. we associate the so-called $Q$-function $Q$ of the c.l.r. $S$ in $\mathfrak{S}$ in the following way. $Q$ is defined on $\rho\left(S^{\circ}\right)$, its values are in [\$S] and it satisfies the relation

$$
\frac{Q(z)-Q(\zeta)^{*}}{z-\bar{\zeta}}=\Gamma_{\zeta}^{*} \Gamma_{z} \quad\left(z, \zeta \in \rho\left(S^{\circ}\right)\right)
$$

It follows (comp. [7]) that $Q$ is defined by (2.3) up to a selfadjoint operator $C \in[\mathbb{S}]$ and has the form

$$
\begin{aligned}
Q(z) & =C+\left(z-x_{0}\right) \Gamma_{z_{0}}^{*} \Gamma_{z_{0}}+\left(z-z_{0}\right)\left(z-\bar{z}_{0}\right) \Gamma_{z_{0}}^{*}(\stackrel{\circ}{S}-z I)^{-1} \Gamma_{z_{0}} \\
& =C-i y_{0} \Gamma_{z_{0}}^{*} \Gamma_{z_{0}}+\left(z-\bar{z}_{0}\right) \Gamma_{z_{0}}^{*} \Gamma_{z}\left(z, z_{0} \in \rho(\stackrel{\leftrightarrow}{S}), z_{0}=x+i y_{0}\right) .
\end{aligned}
$$


Conversely, every function of the form (2.4) satisfies (2.3). Therefore, after choosing a selfadjoint operator $C$ in $\&$ and the mapping $\Gamma_{z_{0}}$ from $(S)$ onto $\mathfrak{N}_{z_{0}}$ the $Q$-function $Q$ is uniquely determined by the pair $(S, S)$, consisting of the symmetric c.l.r. $S$ and one of its canonical selfadjoint extensions $\dot{S}$. Evidently, $Q$ is holomorphic on $\rho\left(S^{\circ}\right)$.

If $S$ and $\dot{S}$ are bounded operators we have

$$
Q(z)=C+\Gamma^{*}(\stackrel{\circ}{S}-z I)^{-1} \Gamma \text {. }
$$

The relation (2.4) evidently implies the following properties of $Q$ :

(i) $Q$ is piecewise holomorphic in $C_{+} \cup C_{-}$and we have $Q(\bar{z})=$ $Q(z)^{*}(\in[\&])$.

(ii) $\operatorname{Im} Q(z) \gg 0$ if $\operatorname{Im} z>0 .{ }^{6}$

We shall prove that these properties are characteristic for $Q$ functions of c.l. relations, (comp. [9]), that is the following theorem holds true.

THEOREM 2.2. A function $Q$ satisfying the conditions (i) and (ii) above is a Q-function of a simple symmetric c.l.r. $S$ in a Hilbert space $\mathfrak{S}_{\mathrm{S}}$. The c.l.r. $S$ and the corresponding canonical selfadjoint extension $\stackrel{S}{S}$ are up to unitary equivalence uniquely determined by $Q$.

Proof. If $\operatorname{dim} B=1$ this theorem immediately follows from [10, $\S 1]$. The proof in the general case given below is close to $[9, \S 3] .^{7}$ With every $z \in C_{+} \cup C_{-}$we associate a symbol $\varepsilon_{z}$ and consider the linear set $\mathbb{R}$ of all finite formal sums $f$

$$
f:=\sum \varepsilon_{z} \xi_{z}, \quad \xi_{z} \in \mathbb{B S} \quad\left(z \in C_{+} \cup C_{-}\right),
$$

where only finitely many elements $\xi_{z}$ are different from zero. If $f, g \in \mathfrak{R}, f=\sum \varepsilon_{z} \xi_{z}, g=\sum \varepsilon_{z} \eta_{z}$ a scalar product $(\cdot, \cdot)$ is defined in $\mathfrak{L}$ by the equation

$$
(f, g):=\sum_{z, \zeta}\left(\frac{Q(z)-Q(\zeta)^{*}}{z-\bar{\zeta}} \xi_{z}, \eta_{\zeta}\right)
$$

Condition (ii) implies that the scalar product is nonnegative, therefore $\mathfrak{\&}$ can be canonically embedded into a Hilbert space $\mathfrak{S}$. In $\mathbb{Z}$ we consider the subset

$$
\mathfrak{D}_{1}:=\left\{f=\sum \varepsilon_{z} \xi_{z} \in \mathbb{R}: \sum \xi_{z}=0\right\}
$$

${ }^{6} A \gg B$ for two bounded operators $A, B$ means that there exists a $\gamma>0$ such that $A-B \geqq \gamma I$.

7 A shorter proof can be given using M. A. NaImark's theorem on the extension of generalized spectral functions. However, we prefer to give a direct proof as this immediately carries over to the general case of a $\Pi_{\kappa}$-space. 
and define on $\mathfrak{D}_{1}$ the operator $S_{1}$

$$
S_{1} f:=\sum z \varepsilon_{z} \xi_{z} \quad \text { if } f=\sum \varepsilon_{z} \xi_{z} \in \mathfrak{D}_{1} .
$$

It is easy to see that the relation

$$
\left(S_{1} f, g\right)=\left(f, S_{1} g\right) \quad\left(f, g \in \mathfrak{D}_{1}\right)
$$

holds true. Fix $z_{0} \in C_{+}$. The continuity of $Q$ in $z_{0}$ implies for arbitrary $\xi \in \mathbb{B s}$

$$
\left(\varepsilon_{z} \xi-\varepsilon_{z_{0}} \xi, \varepsilon_{z} \xi-\varepsilon_{z_{0}} \xi\right) \longrightarrow 0 \text { if } z \longrightarrow z_{0} .
$$

On the other hand, if $\sum \varepsilon_{z} \xi_{z} \in \mathfrak{R}$ and $\xi_{z_{0}}=0$ we have

$$
g:=\sum \frac{\varepsilon_{z} \xi_{z}-\varepsilon_{z_{0}} \xi_{z}}{z-z_{0}} \in \mathfrak{D}_{1},\left(S_{1}-z_{0} I\right) g=\sum \varepsilon_{z} \xi_{z},
$$

hence the range $\left(S_{1}-z_{0} I\right) \mathfrak{D}_{1}$ is the set of all $f=\sum \varepsilon_{z} \xi_{z} \in \mathbb{R}$ with the property $\xi_{z_{0}}=0$. Therefore this range is dense in $\mathbb{R}$ and the same is true for $\left(S_{1}-\bar{z}_{0} I\right) D_{1}$.

If $f \in\left(S_{1}-z_{0} I\right) \mathfrak{D}_{1}$, that is $f=\left(S_{1}-z_{0} I\right) g, g \in \mathfrak{D}_{1}$, we define $U_{1} f:=$ $\left(S_{1}-\bar{z}_{0} I\right) g$. As $\left(S_{1}-z_{0} I\right) g=0$ implies $g=0$ this definition is correct and $U_{1}$ maps the dense set $\left(S_{1}-z_{0} I\right) D_{1}$ onto the dense set $\left(S_{1}-\bar{z}_{0} I\right) \mathfrak{D}_{1}$. It can be easily checked that we have

$$
\left(U_{1} f, U_{1} f^{\prime}\right)=\left(f, f^{\prime}\right) \quad\left(f, f^{\prime} \in\left(S_{1}-z_{0} I\right) \mathfrak{D}_{1}\right)
$$

therefore $U_{1}$ generates a unitary operator $\dot{U}$ in $\mathfrak{H}_{\text {. }}$ Its inverse Cayley transform $S^{\circ}$

$$
\stackrel{\circ}{S}=\left\{\left\{(\stackrel{\circ}{U}-I) g,\left(z_{0} \stackrel{\circ}{U}-\bar{z}_{0} I\right) g\right\}: g \in \mathfrak{S}\right\}
$$

is a selfadjoint c.l.r. by $\S 1.2$. Evidently it is an extension of $S_{1}$. For $z \neq \bar{z}$ we define linear operators $\Gamma_{z}$ from $(8)$ into $\&$ by

$$
\Gamma_{z} \xi=\varepsilon_{z} \xi \quad(\xi \in(\mathbb{S}) \text {. }
$$

Then (2.6) implies

$$
\left(\Gamma_{z} \xi, \Gamma_{\zeta} \eta\right)=\left(\frac{Q(z)-Q(\zeta)^{*}}{z-\bar{\zeta}} \xi, \eta\right) \quad(z \neq \bar{\zeta})
$$

and it is easy to see that $\Gamma_{z}$ is a continuous mapping from (S) into $\mathfrak{S C}$. Its adjoint $\Gamma_{z}^{*}$ is given by

$$
\left(f, \Gamma_{z} \xi\right)=\left(\Gamma_{z}^{*} f, \xi\right) \quad(f \in \mathfrak{S}, \xi \in \mathbb{S}),
$$

and from (2.7) the relation

$$
\Gamma_{\zeta}^{*}\left(\varepsilon_{z} \xi\right)=\Gamma_{\zeta}^{*} \Gamma_{z} \xi=\frac{Q(z)-Q(\zeta)^{*}}{z-\bar{\zeta}} \xi \quad(z \neq \bar{\zeta})
$$


follows. Moreover, if $z \neq z_{0}$ and $\operatorname{Im} z \neq 0$, then $\left(S_{1}-z I\right)\left(\varepsilon_{z} \xi-\varepsilon_{z_{0}} \xi\right)=$ $\left(z-z_{0}\right) \varepsilon_{z_{0}} \xi$ and

$$
\varepsilon_{z} \xi=\varepsilon_{z_{0}} \xi+\left(z-z_{0}\right)\left(S_{1}-z I\right)^{-1} \varepsilon_{z_{0}} \xi=\varepsilon_{z_{0}} \xi+\left(z-z_{0}\right)(\stackrel{\circ}{S}-z I)^{-1} \varepsilon_{z_{0}} \xi .
$$

The operators $\Gamma_{z}$ therefore satisfy the equation

$$
\Gamma_{z}=\left(I+\left(z-z_{0}\right)(\stackrel{\circ}{S}-z I)^{-1}\right) \Gamma_{z_{0}} .
$$

Consider the following (evidently closed) restriction $S$ of $S$ :

$$
S:=\left\{\{f, g\} \in \stackrel{\circ}{S}:\left(g-z_{0} f, \Gamma_{\bar{z}_{0}} \xi\right)=0 \text { for all } \xi \in \mathbb{B}\right\} \text {. }
$$

This definition is independent of $z_{0}$. Indeed, we have for arbitrary $z \neq z_{0}$

$$
\begin{aligned}
\left(g-z f, \Gamma_{\bar{z}} \xi\right)= & \left(g-z_{0} f+\left(z_{0}-z\right) f, \Gamma_{\bar{z}} \xi\right)=\left(g-z_{0} f, \Gamma_{\bar{z}} \xi\right) \\
& +\left(z_{0}-z\right)\left(f, \Gamma_{\bar{z}} \xi\right)
\end{aligned}
$$

and it remains to show that $\left(g-z_{0} f, \Gamma_{\bar{z}} \xi\right)=\left(z-z_{0}\right)\left(f, \Gamma_{\bar{z}} \xi\right)$ for all $\xi \in \mathbb{B},\{f, g\} \in \stackrel{\circ}{\text { S. }}$ Since

$$
\left\{\frac{\varepsilon_{\bar{z}} \xi-\varepsilon_{\bar{z}} \xi}{\bar{z}-\bar{z}_{0}}, \varepsilon_{\bar{z}} \xi\right\} \in \dot{S}-\bar{z}_{0} I=\left(\dot{S}-z_{0} I\right)^{*} \text { and }\left\{f, g-z_{0} f\right\} \in \dot{S}-z_{0} I
$$

we get

$$
\left(f, \varepsilon_{\bar{z}} \xi\right)=\left(g-z_{0} f,\left(\bar{z}-\bar{z}_{0}\right)^{-1}\left(\varepsilon_{\bar{z}} \xi-\varepsilon_{\bar{z}} \xi\right)\right)=\left(g-z_{0} f,\left(\bar{z}-\bar{z}_{0}\right)^{-1} \varepsilon_{\bar{z}} \xi\right)
$$

and the assertion follows.

The symmetric c.l.r. $S$ is simple. Indeed, if $z \neq \bar{z}$ we have $\Re(S-z I)=\left\{g-z f:\left(g-z f, \Gamma_{\bar{z}} \xi\right)=0\right.$ for all $\left.\xi \in \mathbb{S},\{f, g\} \in S\right\}$, hence $h \in \bigcap_{z \neq \bar{z}} \Re(S-z I)$ implies $\left(h, \varepsilon_{\bar{z}} \xi\right)=0$ for all $\xi \in \mathbb{B}, z \neq \bar{z}$. But the elements $\varepsilon_{\bar{z}} \xi, \xi \in \mathbb{S}, z \neq \bar{z}$, generate the space $\mathfrak{S}$ and $h=0$ follows. By $\S 1.3$ the symmetric c.l.r. $S$ is an operator.

It follows from the uniform positivity of $\operatorname{Im} Q$ and the relation (2.7) that the range of $\Gamma_{\bar{z}}$ is closed. Therefore this range is the exact defect space $\Re(S-z I)^{\perp}$, and the first part of the theorem is proved.

Let now $S^{\prime}$ be another simple symmetric c.l.r. and let $\dot{S}^{\prime}$ be a canonical selfadjoint extension of $S^{\prime}$ in $\mathfrak{S}^{\prime}$ such that the $Q$-function of $S^{\prime}$ corresponding to the selfadjoint extension $S^{\prime}$ is $Q$. Then we have

$$
\frac{Q(z)-Q(\zeta)^{*}}{z-\bar{\zeta}}=\Gamma_{\zeta}^{*} \Gamma_{z}=\Gamma_{\zeta}^{*} \Gamma_{z}^{\prime} \quad\left(z, \zeta \in C_{+} \cup C_{-}\right) .
$$

Here $\Gamma_{z}^{\prime}$ denotes a mapping from (S) into $\mathfrak{S}^{\prime}$ satisfying conditions (1) and (2) of $\S 2.1$. Define a transformation $V$ from $\mathfrak{S}^{\prime}$ into $\mathfrak{S}$ by 


$$
V \Gamma_{z}^{\prime} \xi=\Gamma_{z} \xi \quad\left(z \in C_{+} \cup C_{-}, \xi \in \mathbb{S}\right) .
$$

It then follows from (2.8) that $V$ is an isometric operator. The simplicity of $S^{\prime}$ and $S$ implies that its domain and its range are dense in $\mathfrak{S E}^{\prime}$ and $\mathfrak{S}$ resp. Therefore $V$ can be extended to a unitary operator from $\mathfrak{S}^{\prime}$ onto $\mathfrak{S}$. Moreover, we have

$$
V\left(\Gamma_{\zeta}^{\prime}+(z-\zeta)\left(S^{\circ}-z I\right)^{-1} \Gamma_{\zeta}^{\prime}\right)=V \Gamma_{\zeta}^{\prime}+(z-\zeta)\left(S^{\circ}-z I\right)^{-1} V \Gamma_{\zeta}^{\prime},
$$

and $V\left(\dot{S}^{\prime \prime}-z I\right)^{-1}=(\stackrel{S}{S}-z I)^{-1} V$ follows. But this evidently implies that the Cayley transforms $\mathfrak{V}_{z_{0}}\left(\dot{S}^{\prime}\right)$ and $\mathfrak{F}_{z_{0}}\left(S^{\circ}\right)$ are unitarily equivalent. Hence the same holds true for the c.l.r. $\dot{S}^{\prime}$ and $\dot{S}$.

Now $\left\{f^{\prime}, g^{\prime}\right\} \in \dot{S}^{\prime}, V f^{\prime}=f, V g^{\prime}=g,\{f, g\} \in \dot{S}$ yields

$$
\left(g^{\prime}-z f^{\prime}, \Gamma_{\bar{z}}^{\prime} \xi\right)=\left(g-z f, \Gamma_{\bar{z}} \xi\right),
$$

which implies that $V$ establishes a unitary equivalence between $S^{\prime}$ and $S$.

COROLLARY 2.3. A function $Q$ satisfying the conditions (i) and (ii) admits a representation

$$
Q(z)=C+\left(z-x_{0}\right) \Gamma^{*} \Gamma+\left(z-z_{0}\right)\left(z-\bar{z}_{0}\right) \Gamma^{*}(\stackrel{\circ}{S}-z I)^{-1} \Gamma^{\prime}
$$

with a Hilbert space $\mathfrak{S}$, a boundedly invertible operator $\Gamma$ from $\$$ S into $\mathfrak{S}$, a bounded selfadjoint operator $C$ in (S) and a selfadjoint c.l.r. $\stackrel{S}{S}$ in $\mathfrak{S}\left(z_{0} \neq \bar{z}_{0} ; z_{0}=x_{0}+i y_{0}\right)$ with the property

$$
\mathfrak{S}=\text { c.l.s. }\left\{\left(I+\left(z-z_{0}\right)(\dot{S}-z I)^{-1}\right) \Gamma \mathbb{S}: z \neq \bar{z}\right\} \text {. }
$$

Moreover, if a representation

$$
Q(z)=\hat{C}+\left(z-x_{0}\right) \hat{\Gamma}^{*} \hat{\Gamma}+\left(z-z_{0}\right)\left(z-\bar{z}_{0}\right) \hat{\Gamma}^{*}(\hat{S}-z I)^{-1} \hat{\Gamma}
$$

holds with a Hilbert space $\widehat{\mathfrak{S}}$, a boundedly invertible operator $\hat{\Gamma}$ from (S) into $\hat{\mathcal{H}}$, a bounded selfadjoint operator $\hat{C}$ in (S) and a selfadjoint c.l.r. $\hat{S}$ in $\hat{\mathfrak{H}}$, then the subspace

$$
\widehat{\mathfrak{S}}_{1}:=\text { c.l.s. }\left\{\left(I+\left(z-z_{0}\right)(\widehat{S}-z I)^{-1}\right) \hat{\Gamma}(S: z \neq \bar{z}\}\right.
$$

is a reducing subspace for the c.l.r. $\hat{S}$. Furthermore $\left.\widehat{S}\right|_{\hat{\mathfrak{1}}_{1}}$ and $\dot{S}$ are unitarily equivalent.

The (up to unitary equivalence) unique c.l.r. $S^{\circ}$ with properties (2.9) and (2.10) will be denoted by $S_{Q}$.

In the following theorem let $\dot{S}$ be a selfadjoint c.l.r. in $\mathfrak{S}, \Gamma$ a boundedly invertible mapping from a Hilbert space $\mathbb{S}$ into $\mathfrak{S}, C$ a selfadjoint bounded operator in $\mathbb{S}$ and 


$$
\begin{aligned}
Q(z)= & C+\left(z-x_{0}\right) \Gamma^{*} \Gamma+\left(z-z_{0}\right)\left(z-\bar{z}_{0}\right) \Gamma^{*}(\dot{S}-z I)^{-1} \Gamma \\
& \left(z_{0} \neq \bar{z}_{0}, z_{0}=x_{0}+i y_{0}\right) .
\end{aligned}
$$

THEOREM 2.4. Let $\xi \in(5), \xi \neq 0$. Then

(1) $\Gamma \xi \in \overline{\mathfrak{D}\left(S^{\circ}\right)}$ if and only if $\lim _{y \uparrow \infty} y^{-1}(Q(i y) \xi, \xi)=0$,

(2) $\Gamma \xi \notin \mathfrak{D}(\stackrel{S}{)})$ if and only if $\lim _{y \uparrow \infty} y \operatorname{Im}(Q(i y) \xi, \xi)=\infty$.

Proof. (1) The representation (1.6) of $(\dot{S}-z I)^{-1}$ yields

$$
\begin{aligned}
(Q(i y) \xi, \xi)= & y^{-1}\left(\left(i y-x_{0}\right) I+\left(i y-z_{0}\right)\left(i y-\bar{z}_{0}\right)\left(\stackrel{\circ}{S}_{0}-i y I\right)^{-1}\right) \\
& \left.\times P_{0} \Gamma \xi, P_{0} \Gamma \xi\right)+y^{-1}\left(i y-x_{0}\right)\left(\left(I-P_{0}\right) \Gamma \xi,\left(I-P_{0}\right) \Gamma \xi\right) \\
& +y^{-1}(C \xi, \xi)
\end{aligned}
$$

where $S_{0}$ is the (selfadjoint) operator part of $S^{\circ}$ and $P_{0}$ denotes the orthogonal projector onto $\overline{\mathfrak{D}(\dot{S})}$. The arguments in $[9, \S 3]$ imply that the first term on the right hand side tends to zero if $y \uparrow \infty$, the same is obvious for the last term. Therefore

$$
\lim _{y \uparrow \infty} \frac{1}{y}(Q(i y) \xi, \xi)=0 \text { if and only if }\left(I-P_{0}\right) \Gamma \xi=0
$$

and the first statement of the theorem is proved.

(2) Now we start from the identity

$$
\begin{aligned}
& y \operatorname{Im}(Q(i y) \xi, \xi) \\
& \quad=y^{2}\left\|\left(I+\left(i y-z_{0}\right)\left(\dot{S}_{0}-i y I\right)^{-1}\right) P_{0} \Gamma \xi\right\|^{2}+y^{2}\left\|\left(I-P_{0}\right) \Gamma \xi\right\|^{2} .
\end{aligned}
$$

If $\Gamma \xi \notin \overline{\mathscr{D}(\stackrel{S}{S})}$, that is if $\left(I-P_{0}\right) \Gamma \xi \neq 0$ we evidently have

$$
\lim _{y \uparrow \infty} y \operatorname{Im}(Q(i y) \xi, \xi)=\infty \text {. }
$$

If $\Gamma \xi \in \overline{\mathfrak{D}(\dot{S})}=\overline{\mathfrak{D}\left(\dot{S}_{0}\right)}$ we conclude as in [9, Theorem 3.2] that the conditions

$$
\lim _{y \uparrow \infty} y \operatorname{Im}(Q(i y) \xi, \xi)=\infty \quad \text { and } \quad \Gamma \xi \notin \mathfrak{D}(\stackrel{S}{)})
$$

are equivalent. Now (2) easily follows.

CoRollary 2.5. The selfadjoint c.l.r. $S^{\circ}$ in Corollary 2.3 is an operator if and only if the function $Q$ has the property

$$
\lim _{y \uparrow \infty} \frac{1}{y}(Q(i y) \xi, \xi)=0 \text { for all } \xi \in \mathbb{S}, \xi \neq 0 .
$$

Indeed, by the first part of Theorem 2.4 condition (2.11) is equivalent to $\Gamma_{z_{0}}\left(\mathbb{S} \subset \overline{\mathfrak{D}(\dot{S})}\right.$. This yields $\Gamma_{z}(\mathbb{S} \subset \overline{\mathfrak{D}(\dot{S})}$ for all $z \neq \bar{z}$. The subspaces 
$\Gamma_{z}(\mathfrak{S}, z \neq \bar{z}$, generate the space $\mathfrak{S}$ and it follows $\mathfrak{S}=\overline{\mathfrak{D}(\stackrel{S}{S})}$.

CoROLlaRY 2.6. The domain $\mathfrak{D}(S)$ of the simple c.l.r. $S$ in Theorem 2.2 is dense in $\overline{\mathfrak{D}(\dot{S})}$ if and only if $Q$ has the property

$$
\lim _{y \uparrow \infty} y \operatorname{Im}(Q(i y) \xi, \xi)=\infty \text { for all } \xi \in \mathbb{B}, \xi \neq 0 \text {. }
$$

Indeed, $\mathfrak{D}(S)$ is not dense in $\overline{\mathfrak{D}(\grave{S})}$ if and only if there exists an element $\left.f_{0} \in \overline{\mathfrak{D}(\dot{S})}\right), f_{0} \neq 0, f_{0} \perp \mathfrak{D}(S)$. Consider $g_{0}:=\left(\stackrel{\dot{S}}{-}-z_{0} I\right)^{-1} f_{0} \in \mathfrak{D}(\dot{S})$. We have $g_{0} \perp\left(S-\bar{z}_{0} I\right) \mathfrak{D}(S)$, that is $g_{0}=\Gamma_{z_{0}} \xi$ with a certain $\xi \in \mathbb{S}$, $\xi \neq 0$. But by the second part of the theorem such an element $g_{0} \neq 0$ exists if and only if (2.12) does not hold.

3. Generalized resolvents of a symmetric c.1.r. with equal defect numbers. 1. We consider a symmetric c.l.r. $S$ in $\mathfrak{S}$ with equal finite or infinite defect numbers $n_{+}=n_{-}=: n(>0)$. Let the c.l.r. $\widetilde{S}$ in $\tilde{\mathscr{F}} \supset \mathfrak{F E}$ be a selfadjoint extension of $S$. By $\widetilde{P}$ we denote the orthogonal projector of $\widetilde{\mathscr{F}}$ onto $\mathfrak{S}$. The function $z \rightarrow \widetilde{R}_{z}$ :

$$
\widetilde{R}_{z}=\left.\widetilde{P}(\widetilde{S}-z I)^{-1}\right|_{\mathfrak{\$}}
$$

defined for $z \in \rho(\widetilde{S})\left(\supset C_{+} \cup C_{-}\right)$and with values in [S్ ] is called a generalized resolvent of $S$.

A selfadjoint extension $\widetilde{S}$ in $\tilde{\mathfrak{H}}$ of $S$ is called minimal, if

$$
\tilde{\mathfrak{S}}=\tilde{\mathfrak{S}}_{\min }:=\text { c.l.s. }\left\{\left(I+\left(z-z_{0}\right)(\widetilde{S}-z I)^{-1}\right) \mathscr{H}: z \neq \bar{z}\right\} \text {. }
$$

The relation

$$
\begin{aligned}
& \left(\left(I+\left(z-z_{0}\right)(\widetilde{S}-z I)^{-1}\right) f,\left(I+\left(\zeta-z_{0}\right)(\widetilde{S}-\zeta I)^{-1}\right) g\right) \\
& \quad=\left(\left[I+(z-\bar{\zeta})^{-1}\left(\left(z-z_{0}\right)\left(z-\bar{z}_{0}\right) \widetilde{R}_{z}-\left(\bar{\zeta}-z_{0}\right)\left(\bar{\zeta}-\bar{z}_{0}\right) \widetilde{R}_{\bar{\zeta}}\right)\right] f, g\right)
\end{aligned}
$$

implies that there is a one-to-one correspondence between the set of all generalized resolvents and the set of all minimal selfadjoint extensions of $S$, if we only identify unitarily equivalent extensions. Evidently, given an arbitrary selfadjoint extension $\widetilde{S}$ of $S$, such that (3.1) holds, then the minimal extension $\widetilde{S}_{\min }$ in $\widetilde{\mathcal{F}}_{\min }$ corresponding to $\widetilde{R}_{z}$ and the restriction of $\widetilde{S}$ to the reducing subspace

$$
\text { c.l.s. }\left\{\left(I+\left(z-z_{0}\right)(\widetilde{S}-z I)^{-1}\right) \mathfrak{S}: z \neq \bar{z}\right\}
$$

are unitarily equivalent.

Proposition 3.1. If $\widetilde{R}_{z}$ is a generalized resolvent of the symmetric c.l.r. $S$, then the domain of holomorphy of $\widetilde{R}_{z}$ coincides with the resolvent set of the corresponding minimal selfadjoint extension $\widetilde{S}_{\min }$ of $S$. 
Proof. It is obvious that $\widetilde{R}_{z}$ is holomorphic on $\rho\left(\widetilde{S}_{\mathrm{min}}\right)$. Conversely, let $x$ be real and suppose $\widetilde{R}_{z}$ is holomorphic at $z=x$. Choose a circle $C$ with centre $x$, such that $\widetilde{R}_{z}$ is holomorphic on the corresponding closed disc. Then it easily follows from the resolvent identity and Cauchy's integral formula that

$$
\begin{gathered}
\int_{C}\left(\left(\widetilde{S}_{\min }-z I\right)^{-1}\left(I+\left(\zeta-z_{0}\right)\left(\widetilde{S}_{\min }-\zeta I\right)^{-1}\right) f,\right. \\
\left.\left(I+\left(\zeta^{\prime}-z_{0}\right)\left(\widetilde{S}_{\min }-\zeta^{\prime} I\right)^{-1}\right) g\right) d z=0
\end{gathered}
$$

for arbitrary $f, g \in \mathfrak{S}$ and $\zeta, \zeta^{\prime} \in C_{+} \cup C_{-}$outside $C$. As the set of elements of the form $\left(I+\left(\zeta-z_{0}\right)\left(\widetilde{S}_{\min }-\zeta I\right)^{-1}\right) f$ is total in $\widetilde{\mathcal{F}}_{\min }$, the spectral function of $\left(\widetilde{S}_{\min }\right)_{0}$ is constant at $x$, that is $x \in \rho\left(\widetilde{S}_{\min }\right)$.

The generalized resolvent $\widetilde{R}_{z}$ of the symmetric c.l.r. $S$ is called canonical (nonnegative), if the corresponding minimal selfadjoint extension $\widetilde{S}_{\text {min }}$ is canonical, that is $\widetilde{\mathfrak{F}}_{\min }=\tilde{\mathfrak{E}}$ (nonnegative resp.).

2. In order to describe the set of all generalized resolvents of $S$ we fix a canonical selfadjoint extension $\dot{S}$, choose a Hilbert space $(\&)$ with $\operatorname{dim}\left(\mathbb{S}=n\right.$ and construct an operator family $\left(\Gamma_{z}\right), z \neq \bar{z}$, according to (2.1). By $Q$ we again denote a corresponding $Q$-function (see (2.4)). Then the following extension of Krenn's formula for the generalized resolvents of symmetric operators holds true.

THEOREM 3.2. Let $S$ be a symmetric c.l.r. in $\mathfrak{S}$ with equal defect numbers $n>0$. The formula

$$
\widetilde{R}_{z}=(\stackrel{\leftrightarrow}{S}-z I)^{-1}-\Gamma_{z}(Q(z)+\mathscr{T}(z))^{-1} \Gamma_{\frac{z}{z}}^{*}
$$

establishes a one-to-one correspondence between the set of all generalized resolvents $\widetilde{R}_{z}$ of $S$ and the set of all $\mathscr{T} \in \mathfrak{T}(\mathbb{S})$. The generalized resolvent $\widetilde{R}_{z}$ is canonical if and only if $\mathscr{T} \in \mathfrak{I}_{0}(\mathbb{S})$.

Proof. This theorem follows from [8, Theorem 4.1] with the aid of Cayley transformation. Indeed, fix a $z_{0}$ with $\operatorname{Im} z_{0}>0$ and introduce in $\mathfrak{S}$ the isometric operator

$$
U:=\mathbb{E}_{z_{0}}(S)
$$

and its unitary extension

$$
\stackrel{\circ}{U}:=\mathfrak{E}_{z_{0}}(\stackrel{\circ}{)}) .
$$

Then we have $\operatorname{dim} \mathfrak{D}(U)^{\perp}=\operatorname{dim} \mathfrak{R}(U)^{\perp}=n$. We choose $\mathbb{S}^{\prime}:=\mathfrak{D}(U)^{\perp}$ and denote by $\Delta_{0}$ the orthogonal projector onto ('S'. Furthermore define 


$$
\Delta_{\lambda}:=\stackrel{\cup}{U}(\stackrel{\circ}{U}-\lambda I)^{-1} \Delta_{0} \quad(|\lambda| \neq 1), \Delta_{\infty}:=0
$$

and the characteristic function $X$ of the couple $U, U^{\circ}$ by

$$
X(\lambda):=\lambda \Delta_{0} \dot{U}^{*}\left(I-\lambda T^{*}\right)^{-1} \Delta_{0},
$$

where

$$
T f:=\left\{\begin{array}{cl}
U f, & f \in \mathfrak{D}(U) \\
0, & f \in \mathbb{S}^{\prime}
\end{array}\right.
$$

There is a one-to-one correspondence between all unitary extensions $\widetilde{U}$ of $U$ and all selfadjoint extensions $\widetilde{S}$ of $S$, given by $\widetilde{U}=\mathbb{C}_{z_{0}}(\widetilde{S})$. Consider a unitary extension $\widetilde{U}=\mathbb{E}_{z_{0}}(\widetilde{S})$ of $U$ in a Hilbert space $\widetilde{\mathcal{F}} \supset$ $\mathfrak{S}$. The operator-valued function $\tilde{V}$ :

$$
\widetilde{V}_{\lambda}=\left.\widetilde{P}(I-\lambda \widetilde{U})^{-1}\right|_{\mathfrak{s}}
$$

is called a generalized resolvent of $U$. By [8, Theorem 4.1] the formula

$$
\begin{aligned}
\widetilde{V}_{\lambda} & =\left(I-\lambda \stackrel{\circ}{U^{-1}}+\Delta_{1 / \lambda} P(\lambda) \Delta_{\bar{\lambda}}^{*} \quad(|\lambda|<1),\right. \\
P(\lambda): & =(I-E(\lambda))\left(I-X(\bar{\lambda})^{*} \mathscr{E}(\lambda)\right)^{-1}\left(I-X(\bar{\lambda})^{*}\right)
\end{aligned}
$$

establishes a one-to-one correspondence between the set of all generalized resolvents $\widetilde{V}_{\lambda}$ of $U$ and the set of all functions $\mathscr{E}$ with values in [\&'], holomorphic in $\{\lambda:|\lambda|<1\}$ and with the property $\|\mathscr{E}(\lambda)\| \leqq 1(|\lambda|<1)$. Here $\widetilde{V}_{\lambda}$ is canonical (that is $\widetilde{U}$ in (3.3) can be chosen in $\tilde{\mathcal{F}}=\mathfrak{F}$ ) if and only if $\mathscr{E}(\lambda)$ is a unitary operator independent of $\lambda(|\lambda|<1)$.

From the definition of the Cayley transformation and relation (1.4): $\lambda=\left(z-\bar{z}_{0}\right) /\left(z-z_{0}\right)$ it follows

$$
2 i y_{0}\left(I-\lambda^{-1} \widetilde{U}\right)^{-1}=\left(z-\bar{z}_{0}\right) I+\left(z-z_{0}\right)\left(z-\bar{z}_{0}\right)(\widetilde{S}-z I)^{-1}(\operatorname{Im} z>0)
$$

and the corresponding relation between $\dot{S}^{\circ}$ and $\dot{U}^{\circ}$.

Introducing the operator family $\left(\Gamma_{z}^{\prime}\right), z \neq \bar{z}$ :

$$
\Gamma_{\bar{z}_{0}}^{\prime}:=\Delta_{0}, \Gamma_{z}^{\prime}:=\left(I+\left(z-\bar{z}_{0}\right)\left(\dot{S}^{\circ}-z I\right)^{-1}\right) \Gamma_{\bar{z}_{0}}^{\prime}
$$

it is not difficult to see that the operators $\Delta_{\lambda}$ and $\Gamma_{z}^{\prime}$ are connected by the following relations

$$
\Delta_{\lambda}=-\frac{z-z_{0}}{2 i y_{0}} \Gamma_{z}^{\prime}, \Delta_{1 / \bar{\lambda}}^{*}=\frac{z-\bar{z}_{0}}{2 i y_{0}} \Gamma_{\bar{z}}^{\prime *}
$$

Hence (3.4) implies

$$
\widetilde{R}_{z}=(\stackrel{\circ}{S}-z I)^{-1}-\frac{1}{2 i y_{0}} \Gamma_{z}^{\prime} P\left(\lambda^{-1}\right) \Gamma_{z}^{\prime *} \quad(\operatorname{Im} z>0) .
$$


Define a $Q$-function $Q^{\prime}$ of $S$ by

$$
Q^{\prime}(z):=-i y_{0} \Gamma_{z}^{* *} \Gamma_{z_{0}}^{\prime}+\left(z-\bar{z}_{0}\right) \Gamma_{z_{0}}^{*} \Gamma_{z}^{\prime} .
$$

Then we have

$$
\frac{Q^{\prime}(z)-Q^{\prime}(\zeta)^{*}}{z-\bar{\zeta}}=\Gamma_{\zeta}^{*} \Gamma_{z}^{\prime}
$$

and the relation

$$
X\left(\bar{\lambda}^{-1}\right)^{*}=\widetilde{夭}_{-i y_{0}}\left(Q^{\prime}(z)\right) \quad(\operatorname{Im} z>0)
$$

holds. Introducing the c.l.r. $\mathscr{T}^{\prime}(z)$

$$
\mathscr{T}^{\prime}(z):=\left\{\left\{\left(\mathscr{E}\left(\lambda^{-1}\right)-I\right) f,-i y_{0}\left(\mathscr{E}\left(\lambda^{-1}\right)+I\right) f\right\}: f \in \mathbb{S}^{\prime}\right\}
$$

we get

$$
\begin{gathered}
I-X\left(\bar{\lambda}^{-1}\right)^{*}=\left\{\left\{\left(Q^{\prime}(z)+i y_{0} I\right) f, 2 i y_{0} f\right\}: f \in \mathscr{S}^{\prime}\right\} \\
\left(I-X\left(\bar{\lambda}^{-1}\right)^{*} \mathscr{E}\left(\bar{\lambda}^{-1}\right)\right)^{-1}= \\
=\left\{\left\{f_{1}-\left(Q^{\prime}(z)-i y_{0} I\right) f, f_{1}\right\}: \mathscr{E}\left(\lambda^{-1}\right) f_{1}\right. \\
\left.=\left(Q^{\prime}(z)+i y_{0} I\right) f ; f, f_{1} \in \mathscr{S}^{\prime}\right\} \\
P\left(\lambda^{-1}\right)= \\
\left\{\left\{\left(Q^{\prime}(z)+i y_{0} I\right) f_{1}-\left(Q^{\prime}(z)-i y_{0} I\right) \mathscr{E}\left(\lambda^{-1}\right) f_{1},\right.\right. \\
\left.\left.2 i y_{0}\left(I-\mathscr{E}\left(\lambda^{-1}\right)\right) f_{1}\right\}: f_{1} \in \mathscr{S S}^{\prime}\right\}
\end{gathered}
$$

and it is easily seen that this last expression equals $2 i y_{0}\left(Q^{\prime}(z)+\right.$ $\left.\mathscr{T}^{\prime}(z)\right)^{-1}$, that is (3.6) has the form

$$
\widetilde{R}_{z}=\left(S^{\circ}-z I\right)^{-1}-\Gamma_{z}^{\prime}\left(Q^{\prime}(z)+\mathscr{T}^{\prime}(z)\right)^{-1} \Gamma_{\bar{z}}^{\prime *} .
$$

Moreover, (3.8) establishes a one-to-one correspondence between the set of functions $E$ appearing in (3.4) and the set of all $\mathscr{T}^{\prime} \in \mathfrak{I}\left(\mathbb{S}^{\prime}\right)$.

Consider now the Hilbert space $\mathbb{B S}$ and the operator family $\left(\Gamma_{z}\right)$, $z \neq \bar{z}$, appearing in the theorem. Let $J$ denote the partial inverse of $\Gamma_{\overline{z_{0}}}$, that is the mapping from $\mathfrak{N}_{\overline{z_{0}}}=$ (S) $^{\prime}$ onto $(S)$ with the property

$$
J \Gamma_{\overline{z_{0}}}=\left.I\right|_{\propto ⿻} \text {. }
$$

We then have $\Gamma_{z}^{\prime}=\Gamma_{z} J$ and (3.9) implies

$$
\widetilde{R}_{z}=(\dot{S}-z I)^{-1}-\Gamma_{z} J\left(Q^{\prime}(z)+\mathscr{T}^{\prime}(z)\right)^{-1} J^{*} \Gamma_{\bar{z}}^{*} .
$$

Introducing

$$
\mathscr{T}(z):=\left\{\{f, g\}:\left\{J^{-1} f, J^{*} g\right\} \in \mathscr{T}^{\prime}(z)\right\}
$$

and the function $Q: Q(z):=J^{*-1} Q^{\prime}(z) J^{-1}$ we obtain

$$
\frac{Q(z)-Q(\zeta)^{*}}{z-\bar{\zeta}}=J^{*-1} \frac{Q^{\prime}(z)-Q^{\prime}(\zeta)^{*}}{z-\bar{\zeta}} J^{-1}=J^{*-1} \Gamma_{\zeta}^{*} \Gamma_{z}^{\prime} J^{-1}=\Gamma_{\zeta}^{*} \Gamma_{z}
$$




$$
J\left(Q^{\prime}(z)+\mathscr{T}^{\prime}(z)\right)^{-1} J^{*}=(Q(z)+\mathscr{T}(z))^{-1} \cdot
$$

The statement of the theorem now follows without difficulty.

Let us mention that for $z \neq \bar{z}$ it follows from property (ii) in $\S 2.2$ and (1.3) that $(Q(z)+\mathscr{T}(z))^{-1}$ in (3.2) belongs to [S] and that the formula

$$
(Q(z)+\mathscr{T}(z))^{-1}=\left(P_{0} Q(z) P_{0}+\mathscr{T}(z)_{0}\right)^{-1} P_{0}
$$

holds true.

3. Relation (3.2) establishes a one-to-one correspondence between $\mathfrak{T}(\mathbb{S})$ and the minimal selfadjoint extensions $\widetilde{S}$ of the symmetric c.l.r. $S$ if we identify unitarily equivalent extensions. The minimal selfadjoint extension $\widetilde{S}$ corresponding to $\mathscr{T} \in \mathfrak{I}(\mathbb{S})$ will in the following be denoted by $S^{(\tau)} \cdot 8$

Proposition 3.3. Under the conditions of Theorem 3.2 the set $\rho\left(S^{(\mathscr{T})}\right)$ belongs to the domain of holomorphy of $\mathscr{T} \in \mathfrak{I}(\mathbb{S})$.

Proof. Evidently we can take $\mathbb{B S}=\mathbb{S}^{\prime}=\mathfrak{D}(U)^{\perp}$. Then (3.7) implies

$$
\mathscr{E}\left(\lambda(z)^{-1}\right)=I-2 i y_{0}\left(\mathscr{T}^{\prime}(z)+i y_{0} I\right)^{-1} \quad(\operatorname{Im} z>0) .
$$

The function $\mathscr{E}$ extends to $\{\lambda:|\lambda|>1\}$ by

$$
\left.\mathscr{E}(\lambda)=\mathscr{E}\left(\bar{\lambda}^{-1}\right)^{*}\right)^{-1}
$$

(see [8]), hence (3.11) extends to the lower half plane according to

$$
\mathscr{E}(\overline{\lambda(z)})=I-2 i y_{0}\left(\mathscr{T}^{\prime}(\bar{z})+i y_{0} I\right)^{-1} \quad(\operatorname{Im} z \neq 0) .
$$

Here we put $\mathscr{T}^{\prime}(\bar{z})=\mathscr{T}^{\prime}(z)^{*}(\operatorname{Im} z>0)$. The extended function $\mathscr{E}$ is holomorphic in $\{\lambda:|\lambda| \neq 1\}$. Thus it remains to show that $\mathscr{E}$ is holomorphic at $\lambda(x)^{-1}$ if $x$ is a real point in $\rho\left(S^{(\mathscr{\sigma})}\right)$. We observe (see [8, p. 390])

$$
\mathscr{E}\left(\lambda(x)^{-1}\right)=\Delta_{0} \dot{U}^{*} \lambda(x)\left(I-\widetilde{V}_{1 / 2(x)}^{-1}\right) \Delta_{0},
$$

therefore the statement follows if we show that $\widetilde{V}_{1 \mid \lambda(x)}^{-1}$ is holomorphic at $x$. But from (3.5) it follows

$$
2 i y_{0} \widetilde{V}_{1 / \lambda(x)}=\left.\left(x-\bar{z}_{0}\right) \widetilde{P}\left(I+\left(x-z_{0}\right)\left(S^{(\sigma)}-x I\right)^{-1}\right)\right|_{\mathfrak{\xi}},
$$

and it remains to show that the operator on the right hand side has a bounded inverse.

Suppose there exists an element $f_{0} \in \mathfrak{K}$ such that $\Gamma_{z_{0}}$. 


$$
\left(f_{0},\left(I+\left(x-z_{0}\right)\left(S^{(\mathscr{T})}-x I\right)^{-1}\right) f\right)=0 \text { for all } f \in \mathscr{L} \text {. }
$$

Denote $f_{0}^{\prime}:=P_{0} f_{0}$, where $P_{0}$ is the orthoprojector onto $\overline{\mathcal{D}\left(S^{(5)}\right)}$ and let $E_{0}$ be the spectral function of the operator part $S_{0}^{(\mathscr{\sigma})}$ of $S^{(\mathscr{S})}$. Then we have

$$
0=\left(f_{0}^{\prime}, f_{0}^{\prime}+\left(x-z_{0}\right)\left(S_{0}^{(\sigma)}-x I\right)^{-1} f_{0}^{\prime}\right)=\int_{-\infty}^{\infty} \frac{t-z_{0}}{t-x} d\left(E_{0}(t) f_{0}^{\prime}, f_{0}^{\prime}\right) .
$$

Now $x \in \rho\left(S^{(-)}\right)$implies $f_{0}^{\prime}=0$ and from (3.13) we get $f_{0}=0$. Furthermore, suppose there exists a sequence $\left(f_{n}\right)$ in $\mathfrak{S}_{c},\left\|f_{n}\right\|=1$, such that $\widetilde{P}\left(f_{n}+\left(x-z_{0}\right)\left(S^{(\sigma)}-x I\right)^{-1} f_{n}\right) \rightarrow 0$ if $n \rightarrow \infty$. Then a simple modification of the previous argument gives a contradiction.

REMARK. If $S^{(\prime)}$ is a bounded operator it easily follows that $\mathscr{T}$ is holomorphic also at $\infty$, which means that the corresponding function $\mathscr{E}$ from (3.8) is holomorphic at $\lambda=1$.

Proposition 3.4. Suppose the c.l.r. $S$ and $S$ in Theorem 3.2 are bounded linear operators, $S$ is nondensely defined and $\Gamma_{z}, Q$ are chosen according to (2.2) and (2.5) with $C=0$. If the extension $S^{(\mathscr{T})}$ of $S$ is canonical, then the inverse $\mathscr{T}^{-1}$ of the c.l.r. $\mathscr{T} \in \mathfrak{I}_{0}(\mathbb{S})$ is a bounded operator.

Proof. Evidently (3.2) implies that $\Delta(z):=(Q(z)+\mathscr{T})^{-1}$ is a bounded operator in $\mathbb{S}$, which depends holomorphically on $z$ if $z \in$ $\rho\left(S^{(r)}\right) \cap \rho\left(S^{\circ}\right)$ and that

(3.14) $\|\Delta(z)\| \leqq K<\infty$ for all sufficiently by large $|z|$ ( $K$ constant) .

Assume there exist elements $f_{n} \in \mathscr{D}(\mathscr{T}),\left\|f_{n}\right\|=1, n=1,2, \cdots$, such that for some $g_{n} \in \mathscr{T}\left(f_{n}\right)$ we have $\left\|g_{n}\right\| \rightarrow 0(n \rightarrow \infty)$. It follows $f_{n}=$ $\Delta(z)\left(Q(z) f_{n}+g_{n}\right)$ and $1=\left\|\Delta(z)\left(Q(z) f_{n}+g_{n}\right)\right\|$. But if $|z|$ and $n$ are large enough the right hand side is arbitrarily small, contradiction. Therefore $\{f, g\} \in \mathscr{T}$ implies $\|g\| \geqq \gamma\|f\|$ with a certain $\gamma>0$, that is $\mathscr{T}^{-1}$ is a bounded operator.

COROLLARY 3.5. Under the assumptions of Proposition 3.4

$$
\left\|\Delta(x)-\mathscr{T}^{-1}\right\| \longrightarrow 0 \text { if } x \longrightarrow \infty \text {. }
$$

Indeed, if $\mathscr{T}$ is an operator we have

$$
\Delta(x)-\mathscr{T}^{-1}=-\Delta(x) Q(x) \mathscr{T}^{-1},
$$

therefore (3.14) and $\|Q(z)\| \rightarrow 0$ if $z \rightarrow \infty$ imply (3.15). If $\mathscr{T}(0) \neq\{0\}$ denote by $P_{0}$ the orthoprojector onto $\overline{\mathscr{D}(\mathscr{T})}$ and by $\mathscr{T}_{0}$ the operator part of $\mathscr{T}$. Then (comp. (1.8)) 


$$
\left\|\Delta(x)-\mathscr{T}^{-1}\right\|=\left\|\left[\left(P_{0} Q(x) P_{0}+\mathscr{T}_{0}\right)^{-1}-\mathscr{T}_{0}^{-1}\right] P_{0}\right\|
$$

and (3.15) follows in the same way.

4. In [4, Theorem 6.7] a description of the set of all generalized resolvents of a symmetric c.l.r. $S$ with arbitrary (even unequal) defect numbers is given. Here we shall establish the connection between the parameter function $V$ in [4] and the function $\mathscr{T}$ in Theorem 3.2.

We consider the c.l.r. $K_{z}:=R_{z}^{-1}+z I^{9}(\operatorname{Im} z>0)$. Then $V(z)$ in [4] is the restriction

$$
\left.\left(I+2 i y_{0}\left(K_{z}-i y_{0} I\right)^{-1}\right)\right|_{\Re_{z_{0}}} \quad\left(z_{0}=i y_{0}\right),
$$

mapping $\mathfrak{N}_{\bar{z}_{0}}$ into $\mathfrak{R}_{z_{0}}$. On the other hand, using (3.5), it is not difficult to check that this operator coincides with the restriction of $\lambda(z)\left(I-\widetilde{V}_{1 / \lambda(z)}^{-1}\right)$ to $\Re_{\bar{z}_{0}}$. Now (3.12) implies $\mathscr{E}\left(\lambda(z)^{-1}\right)=\dot{U}^{*} V(z)$ or, by (3.7), (3.8) and $\Gamma_{z_{0}}^{* *} \Gamma_{z_{0}}^{\prime}=\left.I\right|_{\Re_{z_{0}}}$,

$$
V(z)=\stackrel{\circ}{U}\left(I-2 i y_{0}\left(Q^{\prime}\left(z_{0}\right)+\mathscr{T}^{\prime}(z)\right)^{-1}\right),
$$

and we finally get from (3.10) and the relation $\dot{U}^{\circ}=\Gamma_{z_{0}}$

$$
\begin{aligned}
& V(z)=\stackrel{\circ}{U}-2 i y_{0} \dot{U} \Gamma_{\bar{z}_{0}}\left(Q\left(z_{0}\right)+\mathscr{T}(z)\right)^{-1} \Gamma_{\bar{z}_{0}}{ }^{*} \\
& =\stackrel{U}{U}-2 i y_{0} \Gamma_{z_{0}}\left(Q\left(z_{0}\right)+\mathscr{T}(z)\right)^{-1} \Gamma_{z_{0}}^{*} .
\end{aligned}
$$

5. In this section $S$ is a simple symmetric c.l.r. in $\mathfrak{F}$ with equal defect numbers and $S$ a fixed canonical selfadjoint extension of $S$. We give a necessary and sufficient condition on $\mathscr{T}$ in order that $S^{(r)}$ is an operator.

Let us remind that with the fixed selfadjoint extension $S$ a $Q$ function $Q$ of $S$ is given by (2.3) or (2.4). Now for each $\mathscr{T} \in \mathfrak{I}(\mathbb{S})$ we define a function $Q_{\mathscr{T}}$ with values in [\&]

$$
Q_{\mathscr{T}}(z):=Q(z)-\left(Q(z)-Q\left(z_{0}\right)^{*}\right)(Q(z)+\mathscr{T}(z))^{-1}\left(Q(z)-Q\left(z_{0}\right)\right) .
$$

It is easy to see that for $\mathscr{T} \in \mathfrak{I}_{0}(\mathbb{S})$ the function $Q$, is a $Q$-function of $S$ corresponding to the canonical selfadjoint extension $S^{(\mathscr{T})}$.

Lemma 3.6. The function $Q_{\sigma}$ in (3.16) has the properties (i) and (ii) of $\S 2.1$.

Proof. Multiplying (3.2) from the left by $\left(z-z_{0}\right)\left(z-\bar{z}_{0}\right) \Gamma_{z_{0}}^{*}$, from the right by $\Gamma_{z_{0}}$ and adding $C+\left(z-x_{0}\right) \Gamma_{z_{0}}^{*} \Gamma_{z_{0}}$ with the operator $C$ from (2.4) we get

$$
Q_{\mathscr{T}}(z)=C+\left(z-x_{0}\right) \Gamma_{z_{0}}^{*} \Gamma_{z_{0}}+\left(z-z_{0}\right)\left(z-\bar{z}_{0}\right) \Gamma_{z_{0}}^{*}\left(S^{(\mathscr{T})}-z I\right)^{-1} \Gamma_{z_{0}},
$$

\footnotetext{
${ }^{8}$ In [4] this c.l.r. is denoted by $T(z)$.
} 
which implies

$$
\begin{aligned}
& \frac{Q_{\mathscr{T}}(z)-Q_{\mathscr{T}}(\zeta)^{*}}{z-\bar{\zeta}} \\
& \quad=\Gamma_{z_{0}}^{*}\left(I+\left(\bar{\zeta}-z_{0}\right)\left(S^{(\mathscr{T})}-\bar{\zeta} I\right)^{-1}\right)\left(I+\left(z-z_{0}\right)\left(S^{(\mathscr{})}-z I\right)^{-1}\right) \Gamma_{z_{0}} .
\end{aligned}
$$

The operator $I+\left(z-z_{0}\right)\left(S^{(\mathscr{T})}-z I\right)^{-1}$ has the bounded inverse operator $I+\left(z_{0}-z\right)\left(S^{(\sigma)}-z_{0} I\right)^{-1}$ and the statement easily follows.

The main result of this section can now be stated.

THEOREM 3.7. The c.l.r. $S^{(\sigma)}$ is an operator if and only if

$$
\lim _{y \uparrow \infty} \frac{1}{y}\left(Q_{\Im}(i y) \xi, \xi\right)=0 \text { for all } \xi \in \mathbb{S} \text {. }
$$

Proof. It follows from Lemma 3.6 and Corollary 2.3 that the function $Q_{\mathscr{T}}$ admits a representation (2.9) with a selfadjoint c.l.r. $S=S_{Q_{\mathscr{S}}}$ in a Hilbert space

$$
\mathfrak{S}_{Q \mathscr{S}}:=\text { c.l.s. }\left\{\left(I+\left(z-z_{0}\right)\left(S_{Q \mathscr{T}}-z I\right)^{-1}\right) \Gamma(S: z \neq \bar{z}\}\right. \text {. }
$$

By Corollary 2.5 $S_{Q_{S}}$ is an operator if and only if (3.18) holds true. On the other hand we have the representation (3.17). Therefore the second part of Corollary 2.3 implies that the subspace

$$
\widehat{\mathfrak{S}}_{1}:=\text { c.l.s. }\left\{\left(I+\left(z-z_{0}\right)\left(S^{(\mathscr{I})}-z I\right)^{-1}\right) \Gamma_{z_{0}}(\&: z \neq \bar{z}\}\right.
$$

is reducing for $S^{(\mathscr{T})}$, and $\left.S^{(\sigma)}\right|_{\hat{\sigma}_{1}}, S_{Q_{\sigma}}$ are unitarily equivalent and can thus be identified.

It remains to show that $S^{(\mathscr{T})}$ is an operator if and only if $S_{Q_{\sigma}}$ is an operator.

If $S^{(\mathscr{\sigma})}$ is an operator then its restriction $S_{Q_{\mathscr{\sigma}}}$ is evidently also an operator. Assume $S^{(F)}(0) \neq\{0\}$ and $S_{Q_{\sigma}}(0)=\{0\}$. The space $\tilde{\mathcal{E}}$ in which $S^{(\sigma)}$ is defined has a decomposition $\tilde{\mathfrak{F}}=\hat{\mathfrak{F}}_{1} \oplus \widehat{\mathscr{S}}$. Let $S^{(\sigma)}=$ $S_{Q_{\mathscr{T}}} \oplus \hat{S}$ be the corresponding decomposition of $S^{(\mathscr{T})}$. The assumption $S^{(\mathscr{F})}(0) \neq\{0\}, \quad S_{Q_{F}}(0)=\{0\}$ implies $S^{(\mathscr{\sigma})}(0)=\hat{S}(0)$, hence $S^{(\mathscr{F})}(0) \perp \hat{\mathcal{F}}_{1}$. We consider an element $\widetilde{f} \in S^{(F)}(0)$. Then $f:=\widetilde{P} \widetilde{f} \perp \Gamma_{z_{0}}(\mathbb{S}$ and

$$
(f, \mathfrak{D}(S))=(\widetilde{P} \tilde{f}, \mathfrak{( S}(S))=(\widetilde{f}, \mathfrak{D}(S))=\{0\},
$$

since $\mathfrak{D}(S) \subset \overline{\mathfrak{D}\left(S^{(\mathscr{T})}\right)}=S^{(\mathscr{T})}(0)^{\perp}$. Therefore we have $f=g_{0}-z_{0} f_{0}$ with a certain $\left\{f_{0}, g_{0}\right\} \in S$, and $\left(g_{0}-z_{0} f_{0}, f_{0}\right)=0$, that is $\left(g_{0}, f_{0}\right)=z_{0}\left(f_{0}, f_{0}\right)$. On the other hand $\left\{f_{0}, g_{0}\right\} \in S$ implies that $\left(g_{0}, f_{0}\right)$ is real, hence $f_{0}=0$. Since $S$ is simple we also have $g_{0}=0$. We conclude $f=0$, hence $\widetilde{f} \in \tilde{\mathcal{H}} \ominus \mathscr{S}$, that is $S^{(\mathscr{T})}(0) \subset \tilde{\mathfrak{F}} \ominus \mathscr{S}$. Now it is easy to see that this is a contradiction to the minimality of $S^{(\mathscr{T})}$. Therefore $S^{(\mathscr{T})}(0) \neq\{0\}$ implies $S_{Q_{\mathscr{S}}}(0) \neq\{0\}$ and the proof is complete. 
REMARK. We do not know if the operators $S_{Q_{\sigma}}$ and $S^{(\sigma)}$ are unitarily equivalent in general. But this is evidently true if $\mathscr{T} \in$ $\mathfrak{T}_{0}(\mathbb{S})$, that is if the extension $S^{(\mathscr{T})}$ is canonical.

4. Generalized resolvents of nondensely defined symmetric contractions. 1. Recently M. G. Kreǐn and I. E. Ovčarenko [11, 12] gave a description of all generalized contraction resolvents of a symmetric nondensely defined contraction in a Hilbert space. In this section we show that such a description can be easily obtained from the "classical" results of M. G. Krein [15] and Theorem 3.2.

Let $S$ be a nondensely defined symmetric closed contraction in $H$, that is $S$ is an operator and we have

$$
(S f, g)=(f, S g),\|S f\| \leqq\|f\| \quad(f, g \in \mathfrak{D}(S)) .
$$

A generalized resolvent $\widetilde{R}_{z}$ of $S$, whose corresponding minimal selfadjoint extension $\widetilde{S}$ of $S$ is a contraction, is called a selfadjoint contraction resolvent (s.c.-resolvent).

By a classical result of M. G. Krein [15] with $S$ there are associated two canonical selfadjoint contraction (c.s.c.-)extensions $S_{\mu}$ and $S_{I I}$ of $S$ such that the inequalities

$$
\text { - }\left(S_{\mu} f, f\right) \leqq(\widetilde{S} f, f) \leqq\left(S_{M} f, f\right) \quad(f \in \mathfrak{S})
$$

hold true for an arbitrary c.s.c.-extension $\widetilde{S}$ of $S$. The extensions $S_{\mu}$ and $S_{M}$ can be characterized in the following way. If $\mathfrak{R}:=\mathfrak{D}(S)^{\perp}$, then for an arbitrary c.s.c.-extension $\widetilde{S}$ we have $\widetilde{S}=S_{\mu}\left(\widetilde{S}=S_{M}\right)$ if and only if

$$
\int_{-1}^{1} \frac{d(\widetilde{E}(\lambda) \varphi, \varphi)}{1+\lambda}=\infty \quad\left(\int_{-1}^{1} \frac{d(\widetilde{E}(\lambda) \varphi, \varphi)}{1-\lambda}=\infty \text { resp. }\right)
$$

for all $\varphi \in \mathfrak{N}, \varphi \neq 0($ see [15, Theorem 4]). Here $\widetilde{E}$ denotes the spectral function of $\widetilde{S}$.

2. Let $(S)$ be a Hilbert space with $\operatorname{dim} \mathbb{S}=\operatorname{dim} \mathfrak{R}, \stackrel{S}{S}=S_{\mu}, \Gamma$ a bijection from $\mathbb{S}$ onto $\mathfrak{N}$ and set

$$
\Gamma_{z}:=\left(S_{\mu}-z I\right)^{-1} \Gamma, Q(z):=\Gamma^{*}\left(S_{\mu}-z I\right)^{-1} \Gamma
$$

(see (2.2), (2.5)). Then the set of all generalized resolvents of $S$ (in the sense of (3.1)) is given by (3.2). Evidently $S^{(\mathscr{T})}=S_{\mu}$ if $\mathscr{T}=$ $\mathscr{T}_{\mu}:=\{\{0, g\}: g \in \mathbb{S}\}$. Define the selfadjoint c.l.r. $\mathscr{T}_{M}$ in $(S)$ by $S^{\left(\mathscr{F}_{M}\right)}=$ $S_{M}$. By Proposition 3.4 $\mathscr{T}_{M}^{-1}$ is a bounded operator. Moreover we have

$$
\mathscr{T}_{M}^{-1}=\Gamma^{-1}\left(S_{M}-S_{\mu}\right) \Gamma^{*-}
$$

Indeed, (3.2) and (4.1) imply ( $x$ real, $|x|>1$ ) 


$$
\begin{aligned}
& \left(S_{M}-x I\right)^{-1}-\left(S_{\mu}-x I\right)^{-1} \\
& \quad=-\left(S_{\mu}-x I\right)^{-1} \Gamma\left(Q(x)+\mathscr{T}_{M}\right)^{-1} \Gamma^{*}\left(S_{\mu}-x I\right)^{-1},
\end{aligned}
$$

hence

$$
\left(S_{\mu}-x I\right)\left(S_{M}-x I\right)^{-1}\left(S_{M}-S_{\mu}\right)=\Gamma\left(Q(x)+\mathscr{T}_{M}\right)^{-1} \Gamma^{*} .
$$

The relation (4.2) now follows from Corollary 3.5 by letting $x \rightarrow \infty$.

By (4.2) the c.l.r. $\mathscr{T}_{M}$ is an operator if and only if the completely indetermined case holds for $S$, which means if and only if $\varphi \in \mathfrak{N} \mid\{0\}$ implies $S_{M} \varphi \neq S_{\mu} \varphi$.

Proposition 4.1. The function $Q$ in (4.1) has the properties

$$
\begin{aligned}
& s-\lim _{x \downarrow 1} Q(x)^{-1}=-\mathscr{T}_{M}^{-1} \\
& s-\lim _{x \uparrow-1} Q(x)^{-1}=0 .{ }^{10}
\end{aligned}
$$

Proof. The existence of the limits on the left hand sides follows from the monotonicity of $Q$, e.g.,

$$
1<x<y \Longrightarrow 0 \ll-Q(x)^{-1} \ll-Q(y)^{-1} \text {. }
$$

For the proof of (4.3) we may evidently assume that $\mathbb{B}=\Re$ and that $\Gamma$ is the embedding operator of $\mathfrak{N}$ into $\mathfrak{K}$. Then it follows from $[15, \S 1.2 . \delta]$ that we have in $\mathfrak{R}$

$$
\left(\Gamma\left(x I-S_{\mu}\right)_{\Re} \Gamma\right)^{-1}=-Q(x),{ }^{11}
$$

that is $-Q(x)^{-1}=\Gamma\left(x I-S_{\mu}\right)_{\Re} \Gamma$. But $x \downarrow 1$ implies $\left(x I-S_{\mu}\right)_{\Re} \downarrow\left(I-S_{\mu}\right)_{\Re}$, and (4.3) follows from the identity $\left(I-S_{\mu}\right)_{\Re}=S_{M}-S_{\mu}$ and (4.2).

To prove (4.4) (see [11, Lemma 2.1]) consider $x<y<-1$ and denote $s-\lim _{y \uparrow-1} Q(y)^{-1}=: K \geqq 0$. Then we have

$$
0 \leqq Q(x)^{1 / 2} Q(y)^{-1} Q(x)^{1 / 2} \leqq I,
$$

therefore $(y \uparrow-1)$

$$
0 \leqq Q^{1 / 2}(x) K Q(x)^{1 / 2} \leqq I,
$$

which implies $0 \leqq K^{1 / 2} Q(x) K^{1 / 2} \leqq I$. On the other hand we have for arbitrary $\phi \in \mathfrak{N}, \phi \neq 0$, with the spectral function $E_{\mu}$ of $S_{l^{\prime}}$

$$
\lim _{x \uparrow-1}(Q(x) \varphi, \varphi)=\int_{-1}^{1} \frac{d\left(E_{\mu}(\lambda) \varphi, \varphi\right)}{1+\lambda}=\infty,
$$

hence $K=0$,

\footnotetext{
${ }_{10} s$-lim denotes the times in the strong operator topology.
}

11 For the definition of $H_{\Re}(H \geqq 0)$ see [15]. 
COROLlaRY 4.2. The following inequalities hold true:

$$
\begin{aligned}
& Q(x)^{-1} \ll \mathscr{T}^{-1} \quad \text { if } \quad x>1, \\
& Q(x)^{-1} \gg 0 \quad \text { if } \quad x<-1 .
\end{aligned}
$$

3. THEOREM 4.3. Let $S$ be a symmetric nondensely defined closed contraction in $\mathfrak{S}, S^{\circ}=S_{\mu}$ and let $\Gamma, Q$ be chosen according to (4.1). Then the relation

$$
\widetilde{R}_{z}=\left(S_{\mu}-z I\right)^{-1}-\Gamma_{z}(Q(z)+\mathscr{T}(z))^{-1} \Gamma^{*}
$$

establishes a one-to-one correspondence between the set of all s.c.resolvents $\widetilde{R}_{z}$ of $S$ and the set of all $\mathscr{T} \in \mathfrak{I}(\mathbb{S})$ with the following properties:

(1) $\mathscr{T}$ is holomorphic in Ext $[-1,1]$ (including $\infty$ ).

(2) If $x \in R^{1} \backslash[-1,1]$, then $0 \leqq \mathscr{T}(x)^{-1} \leqq \mathscr{T}_{M}^{-1}$.

Proof. Suppose $S^{(\mathcal{F})}, \mathscr{T} \in \mathfrak{I}(\mathbb{S})$, is an s.c.-extension of $S$. We consider a real $x_{0},\left|x_{0}\right|>1$, assuming e.g., $x_{0}>1$, and the selfadjoint c.l.r. $\mathscr{T}\left(x_{0}\right)$. According to Theorem 3.2 it generates a canonical selfadjoint extension $S^{\left(\tau\left(x_{0}\right)\right)}$ of $S$, and we evidently have

$$
\left(S^{\left(\sim\left(x_{0}\right)\right)}-x_{0} I\right)^{-1}=\left.\widetilde{P}\left(S^{(\digamma)}-x_{0} I\right)^{-1}\right|_{\tilde{E}} \cdot
$$

The c.l.r. $S^{\left(\tau\left(x_{0}\right)\right)}$ is an operator. Indeed, $\left(S^{\left(\mathscr{T}\left(x_{0}\right)\right)}-x_{0} I\right)^{-1} f_{0}=0$ implies

$$
\left.0=\left(\left(S^{\prime}\right)-x_{0} I\right)^{-1} f_{0}, f_{0}\right) \geqq \frac{1}{x_{0}-1}\left\|f_{0}\right\|^{2},
$$

that is $f_{0}=0$. Now from (4.8) the numerical range of $\left(S^{\left(\mathscr{F}\left(x_{0}\right)\right)}-x_{0} I\right)^{-1}$ is contained in the interval $\left[1 /\left(1+x_{0}\right), 1 /\left(x_{0}-1\right)\right]$, therefore $S^{\left(\left\ulcorner\left(x_{0}\right)\right)\right.}$ is even a contraction and we must have

$$
S_{\mu} \leqq S^{\left(\mathscr{F}\left(x_{0}\right)\right)} \leqq S_{M}
$$

This implies

$$
\left(S_{M}-x I\right)^{-1} \leqq\left(S^{\left(\mathscr{T}\left(x_{0}\right)\right)}-x I\right)^{-1} \leqq\left(S_{\mu}-x I\right)^{-1},
$$

and from (4.9) it follows

$$
\left(Q(x)+\mathscr{T}\left(x_{0}\right)\right)^{-1} \geqq 0,\left(Q(x)+\mathscr{T}\left(x_{0}\right)\right)^{-1} \leqq\left(Q(x)+\mathscr{T}_{M}\right)^{-1} .
$$

Letting $x \rightarrow \infty$ we get $0 \leqq \mathscr{T}\left(x_{0}\right)^{-1} \leqq \mathscr{T}_{M}^{-1}$ by Corollary 3.5 , that is $\mathscr{T} \in \mathfrak{I}(\mathbb{S})$ satisfies condition (2). Condition (1) is satisfied by Proposition 3.3 and the following remark in $\S 3.3$.

Let now $\mathscr{T} \in \mathfrak{T}(\mathbb{S})$ with properties (1) and (2) be given. The Cayley transform 


$$
\mathfrak{E}_{-i}(\mathscr{T})(z)=I-2 i(\mathscr{T}(z)+i I)^{-1} \quad(|z|>1)
$$

can be analytically continued to $z=\infty$ by (1). Moreover for real $z=x,|x|>1$ it follows from

$$
\mathbb{F}_{-i}(\mathscr{T})(x)=-I+2 i\left(i I-\mathscr{T}(x)^{-1}\right)^{-1} \quad(|x|>1)
$$

and (2) that the spectrum of $\mathfrak{E}_{-i}(\mathscr{T})(x)$ has a uniformly positive distance from -1 . Therefore

$$
\mathscr{T}(x)^{-1}=i\left(I-2\left(I+\mathfrak{E}_{-i}(\mathscr{T})(x)\right)^{-1}\right)
$$

is holomorphic in $x,|x|>1$ and the limit

$$
\mathscr{T}(\infty)^{+}:=\lim _{x \rightarrow \infty} \mathscr{T}(x)^{-1}=\lim _{x \rightarrow \infty} i\left(\left(I-2\left(I+\mathfrak{E}_{-i}(\mathscr{T})(x)\right)^{-1}\right)\right.
$$

exists as a bounded operator. Furthermore, as $\|Q(x)\| \rightarrow 0$ if $x \rightarrow \infty$, we have from (1.8)

$$
\lim _{x \rightarrow \infty}(Q(x)+\mathscr{T}(x))^{-1}=\lim _{x \rightarrow \infty} \mathscr{T}(x)_{0}^{-1}\left(P_{0} Q(x) \mathscr{T}(x)_{0}^{-1}+I\right)^{-1} P_{0}=\mathscr{T}(\infty)^{+} .
$$

Now it is easy to see that the c.l.r. $S^{(T)}$ is an operator. Indeed, suppose $\left(S^{(\mathscr{S})}-x I\right)^{-1} f_{0}=0(|x|>1)$. By (4.7)

$$
f_{0}=\Gamma(Q(x)+\mathscr{T}(x))^{-1} \Gamma^{*}\left(S_{\mu}-x I\right)^{-1} f_{0},
$$

and tending $x \rightarrow \infty$ we get $f_{0}=0$.

The proof of the theorem will be complete, if we show that $(Q(x)+\mathscr{T}(x))^{-1}$ is holomorphic for real $x,|x|>1$.

Suppose first that $\mathscr{T}$ is an operator valued function, that is $\mathbb{B}_{\infty}=\{0\}$ in Proposition 1.2. Then

$$
(Q(x)+\mathscr{T}(x))^{-1}=\mathscr{T}(x)^{-1}\left(Q(x)^{-1}+\mathscr{T}(x)^{-1}\right)^{-1} Q(x)^{-1},
$$

and it remains to show that $Q(x)^{-1}+\mathscr{T}(x)^{-1}$ has a bounded inverse if $|x|>1$. But (4.6) and (2) imply

$$
\begin{array}{ll}
Q(x)^{-1}+\mathscr{T}(x)^{-1} \ll-\mathscr{T}_{M}^{-1}+\mathscr{T}(x)^{-1} \leqq 0 & (x>1), \\
Q(x)^{-1}+\mathscr{T}(x)^{-1} \gg 0 & (x<-1),
\end{array}
$$

and the conclusion follows. If $\mathbb{S}_{\infty} \neq\{0\}$ in Proposition 1.2, we have with the orthogonal projector $P_{0}$ onto $\mathbb{S}_{0}$ (see (1.8))

$$
(Q(x)+\mathscr{T}(x))^{-1}=\left(P_{0} Q(x) P_{0}+\mathscr{T}(x)_{0}\right)^{-1} P_{0}
$$

and the same argument can be repeated.

4. Let again $S$ be a symmetric nondensely defined contraction in $\mathfrak{S}$. We consider an interval $[\alpha, \beta]$, where $-\infty<\alpha<-1,1<\beta<\infty$. 
From M.G. Krein's results quoted above it immediately follows that in the set of all canonical selfadjoint bounded extensions $\widetilde{S}$ of $S$ with the property $\sigma(\widetilde{S}) \subset[\alpha, \beta]$ there are two extremal extensions $S_{\mu}^{(\alpha, \beta)}$, and $S_{M}^{(\alpha, \beta)}$, such that for all such extensions $\widetilde{S}$ we have

$$
S_{\mu}^{(\alpha, \beta)} \leqq \widetilde{S} \leqq S_{M}^{(\alpha, \beta)}
$$

Here $S_{\mu}^{(\alpha, \beta)}\left(S_{M}^{(\alpha, \beta)}\right)$ is characterized by the property

$$
\int_{\alpha}^{\beta} \frac{d\left(E_{\mu}(\lambda) f, f\right)}{\lambda-\alpha}=\infty\left(\int_{\alpha}^{\beta} \frac{d\left(E_{M}(\lambda) f, f\right)}{\beta-\lambda}=\infty \text { resp. }\right)
$$

for all $f \in \mathfrak{D}(S)^{\perp}$, where $E_{\mu}\left(E_{M}\right.$ resp.) denotes the spectral function of $S_{\mu}^{(\alpha, \beta)}\left(S_{\mu}^{(\alpha, \beta)}\right.$ resp.). It is evident that $S_{\mu}^{(\alpha, \beta)}=S_{\mu}^{(\alpha, 1)}$ and $S_{M}^{(\alpha, \beta)}=S_{M}^{(-1, \beta)}$. We shall show that these conditions are satisfied, if in (4.7) we put

$$
\mathscr{T}(z) \equiv-Q(\alpha) \quad(\mathscr{T}(z) \equiv-Q(\beta) \text { resp. }) .
$$

Indeed, we have e.g., for $x<\alpha, f \in \mathfrak{S}$

$$
\begin{aligned}
& \left(S^{(-Q(\alpha))}-x I\right)^{-1} f=\left(S_{\mu}-x I\right)^{-1}-\Gamma_{x}(Q(x)-Q(\alpha))^{-1} \Gamma_{x}^{*} f, \\
& \lim _{x \uparrow \alpha}(x-\alpha)\left(\left(S^{(-Q(\alpha))}-x I\right)^{-1} f, f\right)=-\left(Q^{\prime}(\alpha)^{-1} \Gamma_{\alpha}^{*} f, \Gamma_{\alpha}^{*} f\right),
\end{aligned}
$$

and the statement follows if we show that $f \in \mathfrak{D}(S)^{\perp}, f \neq 0$, implies $\Gamma_{\alpha}^{*} f \neq 0$. But from $f_{0} \in \mathscr{D}(S)^{\perp}, \Gamma_{\alpha}^{*} f=0$ we get $f_{0}=(S-\alpha I) g_{0}$ for a certain $g_{0} \in \mathfrak{D}(S)$ and $\left((S-\alpha I) g_{0}, g_{0}\right)=0$, that is $g_{0}=0$ and $f_{0}=0$.

Proposition 4.4. In the set of all selfadjoint extensions of the symmetric nondensely defined contraction $S$ the extension $S_{\mu}^{(\alpha, \beta)}\left(S_{\mu}^{(\alpha, \beta)}\right)$ $i$ is characterized by the following property: $\alpha$ ( $\beta$ resp.) is an isolated eigenvalue of $S_{\mu}^{(\alpha, \beta)}\left(S_{M}^{(\alpha, \beta)}\right.$ resp.) with corresponding eigenspace

$$
\Re(S-\alpha I)^{\perp} \quad\left(\Re(S-\beta I)^{\perp} \text { resp. }\right) .
$$

Proof. We shall only prove the statement about $S_{\mu}^{(\alpha, \beta)}$. Relation (4.5) implies that $(Q(z)-Q(\alpha))^{-1}$ is holomorphic in $z$ in a neighborhood of $\alpha(z \neq \alpha)$. We obtain the corresponding projector $P_{\alpha}$ onto the eigenspace e.g., from (4.11)

$$
P_{\alpha}=-\Gamma_{\alpha} Q^{\prime}(\alpha)^{-1} \Gamma_{\alpha}^{*},
$$

hence $S_{\mu}^{(\alpha, \beta)}$ has the stated property.

Conversely it is easy to see that $x_{n} \in \mathfrak{D}(S), y_{n} \in \Re(S-\alpha I)^{\perp}, x_{n}-y_{n} \rightarrow 0$ $(n \rightarrow \infty)$ implies $x_{n} \rightarrow 0$. Indeed, we have $\left(y_{n},(S-\alpha I) x_{n}\right)=0$ and $\left(x_{n},(S-\alpha I) x_{n}\right)=\left(x_{n}-y_{n},(S-\alpha I) x_{n}\right) \rightarrow 0(n \rightarrow \infty)$, therefore $\left\|x_{n}\right\| \rightarrow$ 0 . This proves that $\mathfrak{D}(S)+\mathfrak{R}(S-\alpha I)^{\perp}$ is a closed subspace of $\mathfrak{S}$. With the argument used above it follows $\mathfrak{S}=\mathfrak{D}(S)+\mathfrak{R}(S-\alpha I)^{\perp}$, 
and it is easy to see that the operator

$$
\widetilde{S} f:= \begin{cases}S f, & f \in \mathfrak{D}(S) \\ \alpha f, & f \in \mathfrak{R}(S-\alpha I)^{-}\end{cases}
$$

is a selfadjoint extension of $S$. Hence $\widetilde{S}=S_{\mu}^{(\alpha, \beta)}$.

These considerations can easily be extended to the case of an infinite interval.

Proposition 4.5. If $S$ is a symmetric nondensely defined contraction in $\mathfrak{S}$ and $-\infty<\alpha \leqq-1$, then there exist two extremal selfadjoint extensions $S_{\mu}^{(\alpha, \infty)}$ : $=S_{\mu}^{(\alpha, 1)}$ and $S_{M}^{(\alpha, \infty)}$ of $S$ such that each canonical selfadjoint c.l.r. extension $\widetilde{S}$ of $S$ with $\widetilde{S} \geqq \alpha I$ has the property

$$
\left(S_{\mu}^{(\alpha, \infty)}-x I\right)^{-1} \geqq(\widetilde{S}-x I)^{-1} \geqq\left(S_{M}^{(\alpha, \infty)}-x I\right)^{-1}
$$

for all $x<\alpha$. The extensions $S_{\mu}^{(\alpha, \infty)}$ and $S_{M}^{(\alpha, \infty)}$ resp. are characterized by the properties

$$
\begin{aligned}
\int_{\alpha}^{\infty} \frac{d\left(E_{\mu}(\lambda) f, f\right)}{\lambda-\alpha} & =\infty \text { for all } f \in \mathfrak{D}(S)^{\perp}, f \neq 0, \text { and } \\
S_{M}^{(\alpha, \infty)}(0) & =\mathfrak{D}(S)^{\perp} \text { resp. },
\end{aligned}
$$

where $E_{\mu}$ denotes the spectral function of $S_{\mu}^{(\alpha, \infty)}$.

Proof. We introduce the transformation $\varphi$

$$
\varphi(S):=I+2(x-\alpha)(S-x I)^{-1} .
$$

Then $\varphi(S)$ is a symmetric contraction, and if $\widetilde{S}$ is a selfadjoint extension of $S, \widetilde{S} \geqq \alpha I$, the operator $\varphi(\widetilde{S})$ is a c.s.c.-extension of $\varphi(S)$. Then

$$
\varphi(S)_{\mu} \leqq \varphi(\widetilde{S}) \leqq \varphi(S)_{M},
$$

and $\varphi(S)_{\mu}$ is characterized by the relation

$$
\begin{aligned}
\int_{-1}^{1} \frac{d\left(F_{\mu}(\lambda) f, f\right)}{\lambda+1}= & \infty \text { for all } f \in \mathfrak{D}(\varphi(S))^{\perp}=\mathfrak{R}(S-x I)^{\perp}, \\
& f \neq 0,
\end{aligned}
$$

where $F_{\mu}$ denotes the spectral function of $\varphi(S)_{\mu}$.

If we define

$$
\begin{aligned}
& S_{\mu}^{(\alpha, \infty)}:=x I+2(x-\alpha)\left(\varphi(S)_{\mu}-I\right)^{-1}, \\
& S_{M}^{(\alpha, \infty)}:=x I+2(x-\alpha)\left(\varphi(S)_{M}-I\right)^{-1},
\end{aligned}
$$

the inequalities (4.12) easily follow from (4.14). Furthermore the 
first condition in (4.13) in equivalent to (4.15) by a simple transformation. Proposition 4.4 implies that $\Re(\varphi(S)-I)^{\perp}$ is the null space of $\varphi(S)_{M}-I$, that is $\Re(\varphi(S)-I)^{\perp}=\mathfrak{D}\left(S_{M}^{(\alpha, \infty)}\right)^{\perp}$. On the other hand $\Re(\varphi(S)-I)^{\perp}=\mathscr{D}(S)^{\perp}$ and the statement follows, since the given characterizations of $S_{\mu}^{(\alpha, \infty)}$ and $S_{M}^{(\alpha, \infty)}$ are independent of $x$. Moreover, the first relations in (4.12) and (4.13) imply

$$
S_{\mu}^{(\alpha, \infty)}=S_{\mu}^{(\alpha, 1)}
$$

CoROLlary 4.6. If $\alpha=-1$ we have $S_{\mu}^{(-1, \infty)}=S_{\mu}$, if $\alpha<-1$ the extension $S_{\mu}^{(\alpha, \infty)}\left(S_{M}^{(\alpha, \infty)}\right)$ corresponds in $(4.7)$ to $\mathscr{T}^{-}(z) \equiv-Q(\alpha)(\mathscr{T}(z) \equiv$ 0 resp.).

The first statement immediately follows from the characterization (4.13) of $S_{\mu^{\prime}}^{(-1, \infty)}$ and the corresponding property of $S_{\mu}$. The statement about $S_{\mu}^{(\alpha, \infty)}, \alpha<-1$, follows from (4.16) and the considerations after (4.12). Finally, put in (4.7) $\mathscr{T}(z) \equiv 0$. It follows

$$
(\widetilde{S}-z I)^{-1} \Gamma=\left(S_{\mu}-z I\right)^{-1} \Gamma-\left(S_{\mu}-z I\right)^{-1} \Gamma Q(z)^{-1} \Gamma^{*}\left(S_{\mu}-z I\right)^{-1} \Gamma=0,
$$

that is $\widetilde{S}(0) \supset \mathfrak{D}(S)^{\perp}$. On the other hand $\widetilde{S}(0)^{\perp}=\overline{\mathfrak{D}(\widetilde{S})} \supset \mathfrak{D}(S)$, hence $\widetilde{S}(0)=\mathscr{D}(S)^{\perp}$. It follows from Proposition 4.5 that $\widetilde{S}=S_{\mu}^{(\alpha, \infty)}$.

5. We shall now generalize the statement of Theorem 4.3. If $S$ is a nondensely defined symmetric contraction with the $Q$-function $Q$ as in Theorem 4.3, we extend the inverse $Q(x)^{-1}$ to $[-\infty, \infty] \backslash(-1,1)$ by defining (in accordance with Proposition 4.1)

$$
Q(x)^{+}:= \begin{cases}Q(x)^{-1}, & x \in(-\infty, \infty) \backslash[-1,1], \\ -\mathscr{S}_{M}^{-1}, & x=1, \\ 0 & , \quad x=-1 \\ +\infty{ }^{11} & , \quad x=-\infty \\ -\infty & , \quad x=+\infty .\end{cases}
$$

Let $-\infty \leqq \alpha \leqq-1,1 \leqq \beta \leqq+\infty$. The generalized resolvent $\widetilde{R}_{z}$ is called an $[\alpha, \beta]$-resolvent if the corresponding minimal selfadjoint extension $\widetilde{S}$ of $S$ has the property

$$
\alpha I \leqq \widetilde{S} \leqq \beta I .
$$

Here, e.g., $-\infty \leqq \widetilde{S} \leqq \beta I, \beta<\infty$ should be read as $\widetilde{S} \leqq \beta I$.

THeOREM 4.7. Let $S, \dot{S}=S_{\mu}, \Gamma, Q$ be as in Theorem 4.3 and suppose $-\infty \leqq \alpha \leqq-1,1 \leqq \beta \leqq \infty$. Then the relation (4.7) establishes $a$ one-to-one correspondence between the set of all $[\alpha, \beta]$-resolvents

${ }^{11}$ Of course the values $\pm \infty$ have only a formal meaning, which will become clear from the following. 
of $S$ and the set of all $\mathscr{T} \in \mathfrak{T}(\mathbb{S})$ with the following properties:

(1) $\mathscr{T}$ is holomorphic in Ext $[\alpha, \beta]$ (including $\infty$ if $\alpha$ and $\beta$ are both finite).

(2) If $x \in(-\infty,+\infty) \backslash[\alpha, \beta]$, then $-Q(\alpha)^{+} \leqq \mathscr{T}(x)^{-1} \leqq-Q(\beta)^{+}$.

Proof. If $\alpha=-1, \beta=1$ this statement coincides with Theorem 4.3. In the proof we shall consider only the case $\alpha=-1, \beta=+\infty$, the other cases being similar.

Suppose $S^{(\sigma)}$ is an extension of $S$ with the property $S^{(\sigma)} \geqq-I$. Choose $x_{0}<-1$ and consider the canonical extension $S^{\left(\mathscr{F}\left(x_{0}\right)\right)}$. The relation

$$
\left(S^{\left(\mathscr{\sigma}\left(x_{0}\right)\right)}-x_{0} I\right)^{-1}=\widetilde{P}\left(S^{(\mathscr{T})}-x_{0} I\right)^{-1}
$$

implies $S^{\left(\mathscr{\sigma}\left(x_{0}\right)\right)} \geqq-I$. By Proposition 4.5

$$
\left(S^{\left(\mathscr{T}\left(x_{0}\right)\right)}-x I\right)^{-1} \leqq\left(S_{\mu}-x I\right)^{-1} \text { for all } x<-1,
$$

hence

$$
\left(Q(x)+\mathscr{T}\left(x_{0}\right)\right)^{-1} \geqq 0 \text { for all } x<-1 .
$$

But we have $Q(x)+\mathscr{T}\left(x_{0}\right)=\left\{\{f, Q(x) f+g\}:\{f, g\} \in \mathscr{T}\left(x_{0}\right)\right\}$, therefore (4.18) implies

$$
(Q(x) f+g, f) \geqq 0 \text { for all }\{f, g\} \in \mathscr{T}\left(x_{0}\right) \text { and } x<-1 .
$$

Letting $x \rightarrow-\infty$ we get $(g, f) \geqq 0$, that is $\mathscr{T}\left(x_{0}\right) \geqq 0$. By Proposition $3.3, \mathscr{T}$ is holomorphic on $(-\infty,-1)$.

Suppose now conversely that a c.l.r. $\mathscr{T} \in \mathfrak{T}(\mathbb{S})$ is given, which is holomorphic in $(-\infty,-1)$ and has the property $\mathscr{T}(x) \geqq 0$ if $x \in$ $(-\infty,-1)$. From $Q(x) \gg 0(x<-1)$ we have $Q(x)+\mathscr{T}(x) \gg 0$, hence $(Q(x)+\mathscr{T}(x))^{-1}$ is an operator. Moreover this operator depends holomorphically on $x$. To show this we may assume that $\mathscr{T}(x)$ is an operator. Then we have

$$
\begin{aligned}
& (Q(x)+\mathscr{T}(x))^{-1} \\
& \quad=(\mathscr{T}(x)+i I)^{-1}\left(I+(Q(x)-i I)(\mathscr{T}(x)+i I)^{-1}\right)^{-1} \quad(x<-1)
\end{aligned}
$$

and since $\mathscr{T}$ is holomorphic in $(-\infty,-1)$ the operator on the right hand side is holomorphic in the same interval. Therefore $\widetilde{P}\left(S^{(\Im)}-z I\right)^{-1}$ is holomorphic in $(-\infty,-1)$, and the statement follows from Proposition 3.1.

6. Let $A$ be a nonnegative c.l.r. in $\mathfrak{F}$. Then the range $\mathfrak{R}(A+I)$ is closed and the inverse $(A+I)^{-1}$ is an operator. Indeed, $\left\{f_{n}, g_{n}\right\} \in$ $A, g_{n}+f_{n} \rightarrow 0(n \rightarrow \infty)$ implies $\left\|f_{n}\right\|^{-1}\left(g_{n}, f_{n}\right)+\left\|f_{n}\right\| \rightarrow 0$. But as $\left(g_{n}, f_{n}\right) \geqq 0$ we get $f_{n} \rightarrow 0, g_{n} \rightarrow 0$ and the conclusion easily follows. 
The c.l.r. $S$

$$
S:=-I+2(A+I)^{-1}
$$

is therefore also an operator and it is easy to see that it is even a (generally nondensely defined) contraction. Moreover, there is a one-to-one correspondence between the set of all nonnegative extensions $\widetilde{A}$ of $A$ and the set of all contractions $\widetilde{S}$, which are extensions of $S$, given by the relation

$$
\widetilde{S}=-I+2(\widetilde{A}+I)^{-1} .
$$

Defining two extremal extensions $A_{M}$ and $A_{\mu}$, corresponding in this way to $S_{M}$ and $S_{\mu}$ resp., M. G. Krein's results quoted above or (4.19) and (4.8) imply the following proposition.

Proposition 4.8. Let $A$ be a nonnegative c.l.r. in $\mathfrak{S}$. Then there exist two nonnegative extensions $A_{\mu}$ and $A_{M}$ of $A$, such that for each nonnegative extension $\tilde{A}$ of $A$ and for arbitrary $x<0$ the inequalities

$$
\left(A_{\mu}-x I\right)^{-1} \leqq \widetilde{P}(\widetilde{A} \cdot x I)^{-1} \leqq\left(A_{M}-x I\right)^{-1}
$$

hold true.

Here $A_{\mu}$ coincides with the Friedrichs' extension of the c.l.r. $A$ introduced in [2]. The relation (4.20) implies

$$
A_{M}(0) \subset \widetilde{A}(0) \subset A_{\mu}(0) .
$$

In order to give a description if all nonnegative extensions of $A$ we may assume that $A=A_{\mu} \cap A_{M}$ holds (otherwise we consider the uniquely determined nonnegative extension $A_{\mu} \cap A_{M}$ of $A$ instead of $A$ ). If in this case $A$ is an operator, then (4.21) implies that $A_{M}$ is also an operator. As was shown in [2], $A_{\mu}$ is an operator if and only if $\mathfrak{D}(A)$ is dense in $\mathfrak{S}$.

Theorem 4.3 implies a description of all nonnegative generalized resolvents of $A$ through the Cayley transformation (4.19). The formulation of this result can be left to the reader. We give here a different description of the nonnegative generalized resolvents of $A$ using a special $Q$-function in the case that the defect numbers of $A$ are finite and equal. This is an immediate generalization of the main result of [15] to the case of c.l.r. In order to get a similar result for arbitrary (including infinite) equal defect numbers a more sophisticated definition of the $Q$-function has to be used (comp. [13]).

Let $A$ be a nonnegative c.l.r. in $\mathfrak{S}$ with finite and equal defect numbers and assume $A=A_{M} \cap A_{\mu}$. By $F_{M}\left(E_{M}\right)$ we denote the spectral function of the operator part $\left(A_{M}\right)_{0}$ of $A_{M}$ (of the maximal extension $S_{M}$ of the Cayley transform $S$ in (4.19) resp.). Then we have from [15] 


$$
\begin{aligned}
& \int_{-1}^{1} \frac{d\left(E_{M}(t) \varphi, \varphi\right)}{1-t}=\infty \text { if } \varphi \in \mathfrak{D}(S)^{\lrcorner}, \varphi \neq 0, \\
& \int_{-1}^{1} \frac{d\left(E_{M}(t) \varphi, \varphi\right)}{t+1}<\infty \text { if } \varphi \in \mathscr{D}(S)^{\perp},
\end{aligned}
$$

and these relations imply

$$
\begin{aligned}
& \int_{0}^{\infty} \frac{1}{\lambda} d\left(F_{M}(\lambda) \varphi, \varphi\right)=\infty \text { if } \varphi \in \Re(A+I)^{\perp}, \varphi \neq 0, \\
& \int_{0}^{\infty} \lambda d\left(F_{M}(\lambda) \varphi, \varphi\right)<\infty \quad \text { if } \quad \varphi \in \Re(A+I)^{\perp} .
\end{aligned}
$$

Hence $\Re(A+I)^{\lrcorner} \subset \mathfrak{D}\left(\left(A_{M}\right)_{0}^{1 / 2}\right)$, and $\left(A_{M}\right)_{0}^{1 / 2} \Gamma_{-1}$ is a bounded operator. Consider the $Q$-function $Q_{0}$ :

$$
Q_{0}(z):=C_{0}+\Gamma_{-1}^{*} \Gamma_{-1}+(z+1) \Gamma_{-1}^{*}\left(I+(z+1)(A-z I)^{-1}\right) \Gamma_{-1}
$$

with

$$
C_{0}:=\left(\left(A_{M}\right)_{0}^{1 / 2} \Gamma_{-1}\right)^{*}\left(\left(A_{M}\right)_{0}^{1 / 2} \Gamma_{-1}\right),
$$

where the operators $\Gamma_{z}$ are defined according to (2.1) with $\AA=A_{M}$. Then we have for $\xi \in \mathbb{B}, \xi \neq 0$

$$
\begin{aligned}
\lim _{x \downarrow-\infty} Q_{0}(x) & =C_{0}+\Gamma_{-1}^{*} \Gamma_{-1}+\lim _{x \downarrow-\infty}(x+1) \int_{0}^{\infty} \frac{\lambda+1}{\lambda-x} d \Gamma_{-1}^{*} F_{M}(\lambda) \Gamma_{-1} \\
& =C_{0}+\Gamma_{-1}^{*} \Gamma_{-1}-\int_{0}^{\infty}(\lambda+1) d \Gamma_{-1}^{*} F_{M}(\lambda) \Gamma_{-1}=0, \\
\lim _{x \uparrow 0}\left(Q_{0}(x) \xi, \xi\right) & =\left(C_{0} \xi, \xi\right)+2\left\|\Gamma_{-1} \xi\right\|^{2}+\int_{0}^{\infty} \frac{1}{\lambda} d\left(\Gamma_{-1}^{*} F_{M}(\lambda) \Gamma_{-1} \xi, \xi\right)=\infty .
\end{aligned}
$$

The proof of the following theorem is similar to the proof of Theorem 4.7, and we omit the details.

THEOREM 4.9. Let $A$ be a nonnegative c.l.r. in $\mathfrak{S}$ with finite and equal defect numbers $n(0<n<\infty)$ and assume $A=A_{M} \cap A_{\mu}$. Then the relation

$$
R_{z}=\left(A_{M}-z I\right)^{-1}-\Gamma_{z}\left(Q_{0}(z)+\mathscr{T}(z)\right)^{-1} \Gamma_{\bar{z}}^{*}
$$

establishes a one-to-one correspondence between the set of all nonnegative generalized resolvents $R_{z}$ of $A$ and the set of all functions $\mathscr{T} \in \mathfrak{I}(\mathbb{S})$ with the following properties:

(1) $\mathscr{T}$ is holomorphic in Ext $[0, \infty)$.

(2) $\mathscr{T}(x) \geqq 0$ if $x<0$.

The canonical nonnegative resolvents of $A$ correspond to $\mathscr{T} \in \mathfrak{I}_{0}(\mathbb{S})$ with property (2), in particular the extension $A_{l}$ is given by $\mathscr{T}(z) \equiv 0$. 
In the case of a linear nonnegative and densely defined operator $A$ with arbitrary defect number $n(\leqq \infty)$ a formula of type (4.22) was given in [13].

\section{REFERENCES}

1. R. Arens, Operational calculus of linear relations, Pacific J. Math., 11 (1961), 9-23.

2. E. A. Coddington, Extension theory of formally normal and symmetric subspaces, Mem. Amer. Math. Soc., 134 (1973).

3. - Spectral theory of ordinary differential operators, Lecture Notes in Mathematics, 448, Springer Verlag, Berlin, 1975.

4. A. Dijksma and H. S. V. de Snoo, Self-adjoint extensions of symmetric subspaces, Pacific J. Math., 54 (1974), 71-100.

5. A. V. Štraus, Extensions and generalized resolvents of a nondensely defined symmetric operator, Izv. Akad. Nauk SSSR, Ser. Mat., 34 (1970), 175-202 (Russian).

6. H. G. Krein, Resolvents of an Hermitian operator with defect index $(m, m)$, Dokl. Akad. Nask SSSR, 52 (1946), 657-660 (Russian).

7. M. G. Kreǐn and H. Langer, Defect subspaces and generalized resolvents of an Hermitian operator in the space $\Pi_{\kappa}$, Functional. Anal. Appl., 5 (1971/1972), 136-146, 217-228. 8. — Über die verallgemeinerten Resolventen und die charakterische Funktion eines isometrischen Operators im Raume $\Pi_{\kappa}$; Colloquia mathematica societatis Janos Bolyai, 5: Hilbert space operators and operator algebras (Tihany 1970), North-Holland Publishing Company, Amsterdam-London, 1972, 353-399.

9. — Über die Q-Funktion eines $\pi$-hermiteschen Operators in Raume $\Pi_{\kappa}$, Acta Sci. Math. (Szeged), 34 (1973), 191-220.

10. - U Über einige Fortsetzungsprobleme, die eng mit der Theorie hermitescher Operatoren in Raume $\Pi_{\kappa}$ zusammenhängen: $I$. Einige Funktionenklassen und ihre Darstellungen. Math. Nachr., 77 (1977), 187-236.

11. M. G. Kreìn and I. E. Ovčarenko, On the Q-functions and the s.c.-resolvents of a nondensely defined Hermitian contraction, to appear (Russian).

12. - On the theory of non-densely defined Hermitian contractions, Dopovidi Akad. Nauk Ukrain, RSR, Ser. A, 10 (1976), 881-884 (Ukrainian).

13. - On generalized resolvents and resolvent matrices of positive Hermitian operators, Dokl. Akad. Nauk SSSR 231, 5 (1976) (Russian).

14. H. Langer and P. Sorjonen, Verallgemeinerte Resolventen hermitescher und isometrischer Operatoren im Pontrjaginraum, Ann. Acad. Sci. Fenn. AI, 561 (1974), 3-45.

15. M. G. Krein, The theory of selfadjoint extensions of semi-bounded Hermitian operators and its applications. I, Mat. Sb., 20 (62) (1947), 431-490 (Russian).

Received September 29, 1976.

TeChnische Universität Dresden, GDR

AND

UNIVERSITY OF LINKÖPING, SWEDEN 



\title{
PACIFIC JOURNAL OF MATHEMATICS
}

\author{
EDITORS
}

RICHARD ARENS (Managing Editor)

University of California

Los Angeles, California 90024

C. W. CurTis

University of Oregon

Eugene, OR 97403

C. C. MOORE

University of California

Berkeley, CA 94720
J. DUgundJI

Department of Mathematics

University of Southern Californı

Los Angeles, California 90007

R. Finn and J. Milgram

Stanford University

Stanford, California 94305

\section{ASSOCIATE EDITORS}

E. F. BECKENBACH

\section{SUPPORTING INSTITUTIONS}

\author{
UNIVERSITY OF BRITISH COLUMBIA \\ CALIFORNIA INSTITUTE OF TECHNOLOGY \\ UNIVERSITY OF CALIFORNIA \\ MONTANA STATE UNIVERSITY \\ UNIVERSITY OF NEVADA, RENO \\ NEW MEXICO STATE UNIVERSITY \\ OREGON STATE UNIVERSITY \\ UNIVERSITY OF OREGON \\ OSAKA UNIVERSITY
}

UNIVERSITY OF SOUTHERN CALIFORNIA

STANFORD UNIVERSITY

UNIVERSITY OF TOKYO

UNIVERSITY OF UTAH

WASHINGTON STATE UNIVERSITY

UNIVERSITY OF WASHINGTON
AMERICAN MATHEMATICAL SOCIETY
NAVAL WEAPONS CENTER 


\section{Pacific Journal of Mathematics \\ Vol. 72 , No. 1 \\ January, 1977}

Kazuo Anzai and Shiro Ishikawa, On common fixed points for several

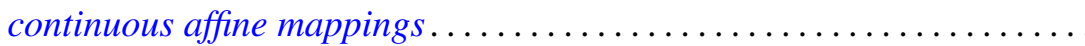

Bruce Alan Barnes, When is a representation of a Banach $*$-algebra

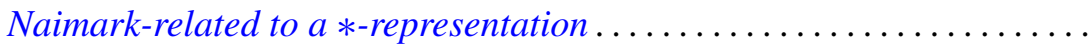

Richard Dowell Byrd, Justin Thomas Lloyd, Franklin D. Pedersen and

James Wilson Stepp, Automorphisms of the semigroup of finite

complexes of a periodic locally cyclic group ...................

Donald S. Coram and Paul Frazier Duvall, Jr., Approximate fibrations and a

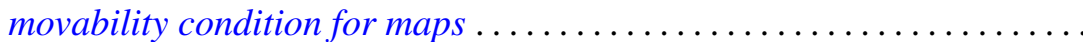

Kenneth R. Davidson and Che-Kao Fong, An operator algebra which is not

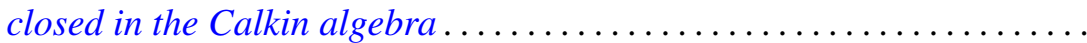

Garret J. Etgen and James Pawlowski, A comparison theorem and oscillation criteria for second order differential systems .............

Philip Palmer Green, $C^{*}$-algebras of transformation groups with smooth

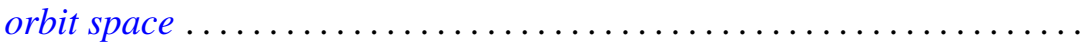

Charles Allen Jones and Charles Dwight Lahr, Weak and norm approximate

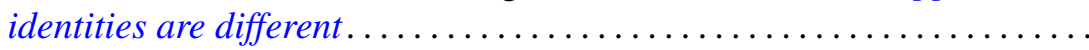

G. K. Kalisch, On integral representations of piecewise holomorphic

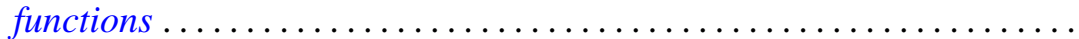

Y. Kodama, On product of shape and a question of Sher

Heinz K. Langer and B. Textorius, On generalized resolvents and

$Q$-functions of symmetric linear relations (subspaces) in Hilbert

space ...................................

Albert Edward Livingston, On the integral means of univalent, meromorphic functions

Wallace Smith Martindale, III and Susan Montgomery, Fixed elements of

Jordan automorphisms of associative rings ..........

R. Kent Nagle, Monotonicity and alternative methods for nonlinear boundary value problems ........................

Richard John O'Malley, Approximately differentiable functions: the $r$ topology.

Mangesh Bhalchandra Rege and Kalathoor Varadarajan, Chain conditions

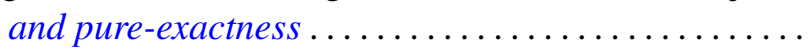

Christine Ann Shannon, The second dual of $C(X)$. .

Sin-ei Takahasi, A characterization for compact central double centralizers of $C^{*}$-algebras .

Theresa Phillips Vaughan, A note on the Jacobi-Perron algorithm. . .

Arthur Anthony Yanushka, A characterization of $\operatorname{PSp}(2 m, q)$ and

$\mathrm{P} \Omega(2 m+1, q)$ as rank 3 permutation groups ......... 